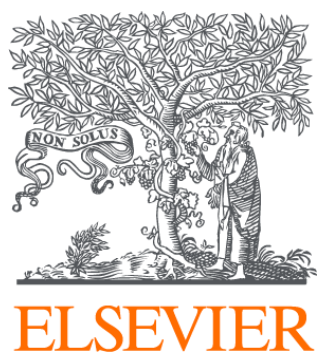

Since January 2020 Elsevier has created a COVID-19 resource centre with free information in English and Mandarin on the novel coronavirus COVID-

19. The COVID-19 resource centre is hosted on Elsevier Connect, the company's public news and information website.

Elsevier hereby grants permission to make all its COVID-19-related research that is available on the COVID-19 resource centre - including this research content - immediately available in PubMed Central and other publicly funded repositories, such as the WHO COVID database with rights for unrestricted research re-use and analyses in any form or by any means with acknowledgement of the original source. These permissions are granted for free by Elsevier for as long as the COVID-19 resource centre remains active. 


\title{
The resilience of Islamic equity funds during COVID-19: Evidence from risk adjusted performance, investment styles and volatility timing
}

\author{
Nawazish Mirza ${ }^{a}$, Syed Kumail Abbas Rizvi ${ }^{c}$, Irum Saba ${ }^{b}$, Bushra Naqvi ${ }^{\text {, }}$ \\ Larisa Yarovaya ${ }^{\mathrm{d}, "}$ \\ ${ }^{\text {a }}$ Excelia Business School, La Rochelle, France \\ ${ }^{\mathrm{b}}$ Institute of Business Administration, Karachi, Pakistan \\ ${ }^{\mathrm{c}}$ Lahore University of Management Sciences, Lahore, Pakistan \\ ${ }^{\mathrm{d}}$ Southampton Business School Southampton, United Kingdom
}

\section{A R T I C L E I N F O}

\section{JEL classification:}

G10

G11

G14

Keywords:

COVID-19

Islamic equity funds

Style analysis

Volatility timing

Resilience

\begin{abstract}
A B S T R A C T
This paper analyses the risk-adjusted performance of Islamic and conventional equity funds during the COVID-19 pandemic. We show that Islamic equity funds demonstrated differentials in risk-adjusted performance, investment styles, and volatility timing compared to their conventional counterparts. Specifically, the results revealed that Islamic equity funds are more resilient to COVID-19 shock since they outperformed non-Islamic peers during the peak months of the pandemic. The trend continues even when the spread smoothens. These findings confirm the safehaven properties of Islamic equity funds, which is helpful for investors aiming to hedge pandemic risks. The style analysis reveals investment drift from riskier styles to more prudent options in response to each stage's uncertainties. The results suggest policymakers should further investigate Islamic financial assets and their underlying principles to improve the resilience of economic systems in any future black swan events.
\end{abstract}

\section{Introduction}

The Coronavirus pandemic (COVID-19) is a global health and societal emergency that had devastating effects on business and finance worldwide. The COVID-19 crisis has economic consequences comparable with the Great Depression and the Global Financial crisis. For a rapidly growing $\$ 2.4$ trillion Islamic Financial industry that currently involves around 1400 institutions spreading across 80 countries, the outbreak's impact is not negligible. On the one hand, some Islamic financial markets and instruments demonstrated safe haven properties during the previous crises (e.g., Hkiri et al., 2017; Foglie \& Panetta, 2020). On the flip side, the early evidence from the COVID-19 pandemic shows that Islamic equity markets have not been entirely immune from this contagious shock (e.g., Yarovaya et al., 2020a).

The Islamic finance literature is extensive. Several studies report that Sharia-compliant products and funds, in general, have demonstrated better performance compared to their conventional counterparts during the turbulent periods (e.g., Ashraf, 2013;

\footnotetext{
* Corresponding author.

E-mail addresses: elahimn@excelia-group.com (N. Mirza), kumail.rizvi@lums.edu.pk (S.K. Abbas Rizvi), isaba@iba.edu.pk (I. Saba), bushra. naqvi@lums.edu.pk (B. Naqvi), L.Yarovaya@soton.ac.uk (L. Yarovaya).
} 
Hoepner et al., 2011; Alqahtani et al., 2017; Boo et al., 2017; Asmild et al., 2019; Alexakis et al., 2019; Safiullah, 2020). The earlier crisis triggered by imprudent lending practices, poor risk management, and accounting malpractices did not similarly affect Islamic finance since many practices are not permitted under Shariah laws. In the context of the Islamic financial system, Islamic mutual funds have an essential role to play.

While both Islamic and conventional funds are investment vehicles, there are certain differentiating factors. The Islamic funds' management and investments need to comply with the Sharia principles. A supervisory board is usually present to monitor and certify such compliance. The stock screening process of Islamic funds requires "income cleansing". This is to ensure that the income streams of the invested companies conform with the standards of Islamic investments. For conventional funds, income cleansing is not obligatory. Another significant differentiation is that while traditional fund managers may or may not include capital structure as their selection criteria, Islamic funds are prohibited from investing in companies with higher gearing levels. It is important to note that investing in Islamic funds is not restricted only to faith-based investors.

It can be argued that Islamic funds perform better in countries with a predominantly Muslim population. Although there can be more investment avenues for the Islamic funds in such countries, the notion of Muslim countries was debunked by Reddy et al. (2017) and Uddin et al. (2018). They provide evidence that Sharia-compliant funds and stocks perform better than conventional alternatives, even in the non-Muslim majority states. The performance of Islamic funds is a function of their investing principles, screening process, investment styles, and timing. Hoepner et al. (2011) documented size-related preferences in investment styles, while Alam et al. (2016) focused on Islamic funds' sectoral inclination (Hayat \& Kraeussl, 2011). suggested that Islamic funds underperform the equity benchmarks. They proposed that index-tracking funds are better options than individual equity funds. We believe that these particular results could be time-specific, and it is interesting to evaluate how Islamic funds are doing after almost a decade of this study.

Furthermore, Tee et al. (2019) and Cheema and Nartea (2018) suggest that these funds might benefit from momentum returns. There are many papers focused on conventional funds; for example, Cao et al. (2017) and Sha (2020) indicate style drifts in response to market conditions, while Rizvi et al. (2020) report the existence of these drifts in the context of the COVID-19 outbreak. However, the possible style drifts in Islamic funds have not been analyzed yet, and we believe it is imperative to evaluate these during the recent global pandemic. The unprecedented changes in market conditions brought by the COVID-19 crisis provide a remarkable opportunity for this assessment. In terms of risk (Bellalah \& Chayeh, 2015), posit that the unique screening criteria of Islamic funds result in a distinguished risk profile. This motivates us to analyze the volatility timing ability of Islamic fund managers.

Specifically, in this paper, we aim to answer the following questions. Do Islamic funds outperform their conventional counterparts during the COVID-19 pandemic? Whether the resilience of Islamic equity funds to the pandemic is determined by the investment styles and effective volatility timing? Our empirical assessment comprises three steps. First, we use traditional measures to evaluate the riskadjusted performance of the Islamic equity funds across six countries, i.e., Malaysia, Pakistan, Saudi Arabia, Qatar, Kuwait, and the UAE. Second, we analyze the stochastic dominance and abnormal returns and perform style analysis to understand the Islamic vs. conventional funds' investment preferences during this outbreak. Third, we document the volatility timing of the two categories of Islamic and conventional equity funds. To observe the impact of the evolution of COVID-19, we segregate our assessment into six stages (between January 2020 and May 2021. The analysis of 1067 equity funds shows that during the outbreak of COVID-19, Islamic funds outperform their conventional peers. We observe a drift in investment styles and evidence of volatility timing for the Islamic funds mainly missing in their conventional counterparts. The results remained robust for the various stages of the outbreak. These findings align with the earlier observations, demonstrating that Islamic funds are resilient during the financial crisis. Our evidence is helpful from a faith-based investor perspective and can also be relevant for investing landscape to consider Islamic funds for optimal portfolio allocations.

This paper also contributes to the growing body of the COVID-19 literature. Some early evidence provided by Zhang et al. (2020), Corbet et al. (2020), Rizvi et al. (2020), Akhataruzzaman et al. (2020), Sharif et al. (2020), Mirza et al. (2020), and Zaremba et al. (2020) have extensively documented the impact of this outbreak on various financial assets and products. However, only a few papers analyzed the effect of this outbreak on Islamic finance (e.g., Aziz et al., 2020; Yarovaya et al., 2020a). This research is the first attempt to provide some initial assessment of conventional vs. Islamic equity funds during the COVID-19 outbreak. The remainder of the paper is organized as follows. Section 2 describes our data and methodology. Section 3 discussed the empirical results and practical implications, and Section 4 concludes.

Table 1

Covid-19 stages for funds' comparison.

\begin{tabular}{|c|c|c|c|c|c|c|}
\hline Year & Stage & Period & Total Cases & Total Deaths & New Cases & New Death \\
\hline 2020 & Stage 1 & January 1 - March 19 & 317201 & 10196 & 317201 & 10196 \\
\hline 2020 & Stage 2 & March 20 - May 11 & 4436288 & 308936 & 4119087 & 298740 \\
\hline 2020 & Stage 3 & May 12 - June 14 & 8295482 & 483383 & 3859194 & 174447 \\
\hline 2020 & Stage 4 & June 15 - September 1 & 26534561 & 942270 & 18239079 & 458887 \\
\hline 2020 & Stage 5 & September 2 - December 31 & 83876749 & 1920805 & 57342188 & 978535 \\
\hline 2021 & Stage 6 & January 1 - May 31 & 171477735 & 3684156 & 87600986 & 1763351 \\
\hline
\end{tabular}

Source: https://www.worldometers.info/coronavirus/as on June 5, 2021

Total Cases and Total Deaths are Cumulative as of the last date during each stage. 


\section{Data and research methodology}

The spread of the COVID-19 has been very sporadic, with limited infections outside China during the first few months. From March 2020 onwards, the virus spread exponentially across Europe, North America, Latin America, the Middle East, and South Asia. This forced stringent state interventions, including lockdowns resulting in moderately flattening the curve momentarily. As the COVID-19 evolved across the globe, economic regression intensified. This continued throughout 2020, and it was only at the end of the year (and beginning 2021) when there was some hope owing to the successful trials of possible vaccines. However, the global vaccination campaigns are very staggered, and eliminating the pandemic will take some time. Given the economic and market consequences of COVID-19, it is interesting to evaluate the comparative performance of funds that are differentiable based on their investment philosophies. This paper considers a period from January 1st' 2020, to May 31st' 2021 that covers this outbreak till very recently. We further split our sample into several subsamples following Rizvi et al. (2020) and identifies six stages of the pandemic. The details of these stages are presented in Table 1.

While it is crucial to assess the comparative funds' performance for the entire period, further analysis of the six stages of the first wave of the COVID-19 can help establish the robustness of our results and provide more detailed results for future research in this area. The statistics from Table 1 show the evolution of total infections and mortality stage-wise for our sample period.

Islamic funds are not very symmetric across the globe, and the main concentration is in Muslim-populated countries. Therefore, we select the top six locations where Islamic funds are domiciled to proceed with the analysis. These include Malaysia, Pakistan, Saudi Arabia, Qatar, Kuwait, and the UAE. For sample selection, we impose two conditions to ensure that only funds that were stable preCOVID are selected. First, we identified funds initiated before June 2018 and existed for at least 18 months before the outbreak. Second, we only consider funds that have reported the positive net asset value (NAV) for 2019. We believe that these criteria are essential to ensure that our results capture the impact of the COVID-19 outbreak. We utilize a sample of 476 Islamic and 591 conventional equity funds, which is sufficient to capture the difference in the performance. We also analyze the allocation of assets under management for potential issues related to cross-border investments. However, we observe that the selected conventional and Islamic funds have sufficiently large investments within their country of domicile. Hence, we do not expect any significant variance to our results from cross-country investments. Although Hoepner et al. (2011) and (Uddin et al., 2019) indicated that Islamic funds prefer to invest globally, we believe that this could be true for the funds domiciled in a location where there are limited Sharia-compliant options. As our sample is specifically from the countries where Islamic finance is very significant, the funds may not need to diversify across borders and incur additional transactional costs.

Furthermore, owing to the better performance of local markets in Malaysia and Pakistan and the conflict within GCC, the funds consolidated their investments locally. These reasons might explain why our selected funds have a substantial investment profile in their domestic markets. The country-wise sample distribution is presented in Table 2.

The intraday NAVs for these funds are collected along with other variables that we explain below. We calculate NAV based daily returns as follows:

$$
R_{i}=\frac{N A V_{t}-N A V_{t-1}}{N A V_{t-1}}
$$

We perform a comparative assessment of Islamic and conventional equity funds using multiple methods. These are discussed below.

\subsection{Risk-adjusted performance during the outbreak}

To assess the risk-adjusted performance, we use three different measures. The first one is the adjusted Sharpe ratio based on Sharpe (1966) and modified by Pezier and White (2006). The modified version does not assume the normality of underlying returns and better suited for data periods with extreme volatility, such as the COVID-19 outbreak. Following Rizvi et al. (2020), we use the following functional form of the adjusted Sharpe ratio $(\gamma)$.

$$
\gamma_{i}=S R_{i}\left(1+\frac{s_{k}}{6} \times S R_{i}-\left(\frac{k_{r}-3}{24}\right)\right) \times S R_{i}^{2}
$$

$S R$ corresponds to the Sharpe ratio, $\mathrm{s}_{\mathrm{k}}$ is skewness while $\mathrm{k}_{\mathrm{r}}$ represents kurtosis. The ratio compares a fund's excess return with the total risk and adjusts for skewness and kurtosis.

The second metric that we used is the Sortino ratio that is based on Sortino and Price (1994) and compares the fund's excess return

Table 2

Sample distribution.

\begin{tabular}{llllll}
\hline Country & Islamic & Conventional & Total & \% AUM within Country - Islamic & \% AUM within Country - Conventional \\
\hline Malaysia & 170 & 207 & 377 & $85 \%$ & $90 \%$ \\
Pakistan & 70 & 85 & 155 & $93 \%$ & $89 \%$ \\
Saudi Arabia & 80 & 103 & 183 & $86 \%$ & $87 \%$ \\
Qatar & 69 & 82 & 151 & $89 \%$ & $90 \%$ \\
Kuwait & 52 & 69 & 121 & $86 \%$ & $91 \%$ \\
UAE & 35 & 45 & 80 & $90 \%$ & \\
Total & $\mathbf{4 7 6}$ & $\mathbf{5 9 1}$ & $\mathbf{1 0 6 7}$ & & \\
\hline
\end{tabular}


with the downside risk. Damianov and Elsayed (2020) noted that the Sortino ratio adequately reflects the risk-adjusted performance of portfolios with turbulent returns.

$$
S=\frac{R_{i}-R_{f}}{\sigma_{d}}
$$

where $\sigma_{\mathrm{d}}$ corresponds to the downside standard deviation.

The third measure we used for risk-adjusted performance is Jensen's alpha ( $\alpha$ ) (Jensen, 1968). This is calculated using the CAPM framework and represented as follows.

$$
R_{i}-R_{f}=\alpha_{i}+\beta_{i}\left(R_{m}-R_{f}\right)+e_{i t}
$$

We proxy the risk-free rate $\left(\mathrm{R}_{\mathrm{f}}\right)$ using the yield on short-term government bonds for each country, while the market risk premium is based on the local representative market index. The country-wise choice of the market index and the risk-free rate is presented in Table 3. The adjusted Sharpe, Sortino, and Jensen's alpha are estimated for Islamic and conventional funds for the entire period and each stage of the outbreak.

\subsection{Stochastic dominance of Islamic Funds}

The second method we use is the stochastic dominance approach (SD). As argued by Vinod (2004), Wong et al. (2008), and Al-Khazali et al. (2014), the SD does not require funds' returns to be normally distributed and can help in ranking the funds without making an assumption about the distribution. In essence, the SD relies on the probability density function of conventional and Islamic funds' returns. We express the returns of Islmic (I) and Conventional funds as fi(x), $\mathrm{fc}(\mathrm{x})$ having a cumulative distribution function of Fi $(\mathrm{x}), \mathrm{Fc}(\mathrm{x})$. The Islamic funds will dominate (first-order) conventional funds if $\mathrm{Fi}(\mathrm{x})-\mathrm{Fc}(\mathrm{x}) \leq 0$. This relationship will suggest that the performance utility of Islamic funds is superior to their conventional peers. Similarly, second-order dominance will require $\int F i(x)-\int F c$ $(x) \leq 0$, while the third order SD will be present if $\iint F i(x)-\iint F \mathrm{c}(x) \leq 0$. Lastly, if $\iiint F i(x)-\iiint F \mathrm{c}(x) \leq 0$, this will represent fourth order dominance. In order to test for the four SD of Islamic and conventional funds, we employ Vinod (2004) methodology that is based on unconventional utility theory.

\subsection{Comparative abnormal returns - Islamic vs. conventional funds}

To substantiate Islamic funds' performance during the COVID-19 pandemic, we extended our data and included the pre-COVID period from January 1 st to December 31, 2019. Therefore, we calculate the abnormal returns for both Islamic and conventional funds by differentiating between pre and post-COVID periods. To estimate the abnormal returns, we follow the event study methodology employed previously by Yarovaya et al. (2020b) and Mirza et al. (2020) with the following specifications of GARCH (1,1).

$$
\begin{aligned}
& R_{i t}=\alpha_{i}+\beta_{i}\left(R_{m t}-R_{f t}\right)+\tau_{i} D_{i t}+\partial_{i} O D_{i t}+\varphi_{i} h_{i t}+e_{i t} \quad \text { with } e_{i} \sim \mathrm{t}_{\mathrm{n}}\left(0, h_{i}\right) \\
& h_{i t}=c_{i}+a_{i} e_{i t-1}^{2}+b_{i} h_{i t-1}+\delta_{i} D_{i t}
\end{aligned}
$$

where $R_{i t}$ corresponds to the fund return, $R_{m t}$ is market return, $R_{f t}$ is the risk-free rate. The dummy $D_{i t}$ takes $\mathrm{t}=1$; if $\mathrm{t}$ is during the COVID period, $h_{i t}$ is conditional variance, and $e_{i t}$ is random error. There are four countries (Kuwait, Saudi Arabia, Qatar, and UAE) in our sample where the oil to GDP rent exceeds $20 \%$. To control this, we further introduce a dummy OD that assumes a value of 1 if the fund is from one of the oil-dependent countries and 0 otherwise. The parameters are represented as $\alpha_{i}, \beta_{i}, \varphi_{i}, c_{i}, a_{i}, b_{i}$, and $\delta_{i}$ that corresponds to the errors in variables. The loading $\tau_{i}$ captures the cumulative abnormal returns. We estimate CARs for the entire period as well as for each of the six stages.

\subsection{COVID-19 and investment styles}

The style analysis is central to assessing returns' sensitivity to various investment classes, strategies, and sectors. The investment styles reflect a fund's manager investment strategy to choose asset classes of different characteristics, which is important during financial turbulence. The differentiation can be based on capitalization (large vs. small), value, growth or momentum, sectoral factors

Table 3

Market Indices and Risk Free for selected Countries.

\begin{tabular}{lll}
\hline & Market Index & Risk Free Rate \\
\hline Malaysia & FTSE Bursa Malaysia Index & Malaysian Govt Bond 10 Years \\
Pakistan & KSE 100 & Pakistan Govt Bond 10 Years \\
Saudi Arabia & Tadawul All Share Index & KSA Govt Bond 10 Years \\
Qatar & QE Index & Qatar Govt Bond 10 Years \\
Kuwait & BKA All Share Index & Kuwait Govt Bond 10 Years \\
UAE & ADX General & UAE Govt Bond 10 Years \\
\hline
\end{tabular}


(cyclical vs. non-cyclical), etc. The changing market conditions may warrant a variation in investment styles for actively managed funds. Cao et al. (2017) and Sha (2020) suggest that performing fund managers can utilize different investment styles to adapt to the changing market conditions. The style analysis helps assess the fund's manager's preferences across various sectors Parikh (2019) and various characteristics Kaiser (2020). We use the standard factorial approach of Sharpe (1992), the return for each fund ( $\left.\mathrm{R}_{\mathrm{i}, \mathrm{t}}\right)$ is expressed as

Table 4

Risk Adjusted Performance Islamic vs. Conventional Funds.

\begin{tabular}{|c|c|c|c|c|c|c|c|c|c|c|c|c|}
\hline \multirow{3}{*}{$\begin{array}{l}\text { Complete Period (Jan 1, } 2020 \text { - May 31, } \\
\text { 2021) } \\
\text { Malaysia }\end{array}$} & \multicolumn{4}{|c|}{ Adjusted Share Ratio } & \multicolumn{4}{|c|}{ Sortino Ratio } & \multicolumn{4}{|c|}{ Jensen's Alpha } \\
\hline & \multicolumn{2}{|l|}{ Islamic } & \multicolumn{2}{|l|}{ Conv. } & \multicolumn{2}{|l|}{ Islamic } & \multicolumn{2}{|l|}{ Conv. } & \multicolumn{2}{|l|}{ Islamic } & \multicolumn{2}{|l|}{ Conv. } \\
\hline & 0.7326 & $* *$ & -0.1200 & $* *$ & 0.5799 & $* *$ & -0.0170 & $* *$ & 0.0283 & $* *$ & -0.0273 & $* * *$ \\
\hline Pakistan & 0.5576 & $* * *$ & -0.3566 & $* *$ & 0.3641 & $* * *$ & -0.1589 & $* * *$ & 0.0568 & $* *$ & -0.0122 & $* *$ \\
\hline Saudi Arabia & 0.6453 & $* *$ & -0.0191 & $* *$ & 0.6274 & $* *$ & -0.0163 & $* *$ & 0.0246 & $* *$ & -0.0514 & $* *$ \\
\hline Qatar & 0.4811 & $* *$ & 0.0155 & $* * *$ & 0.3539 & $* *$ & 0.0035 & $* * *$ & 0.0374 & $* *$ & -0.0289 & $* *$ \\
\hline Kuwait & 0.5684 & $* *$ & 0.1496 & $* * *$ & 0.2819 & $* *$ & 0.0465 & $* * *$ & 0.0515 & $* *$ & -0.0105 & $* *$ \\
\hline \multirow[t]{2}{*}{ UAE } & 0.3811 & $* *$ & 0.1391 & $* * *$ & 0.2601 & $* *$ & 0.0073 & $* *$ & 0.0509 & $* *$ & 0.0128 & $* *$ \\
\hline & \multicolumn{4}{|c|}{ Adjusted Share Ratio } & \multicolumn{4}{|c|}{ Sortino Ratio } & \multicolumn{4}{|c|}{ Jensen's Alpha } \\
\hline Malaysia & $\begin{array}{c}\text { Islamic } \\
0.9783\end{array}$ & $* *$ & $\begin{array}{l}\text { Conv. } \\
0.0266\end{array}$ & $* *$ & $\begin{array}{c}\text { Islamic } \\
0.7975\end{array}$ & $* *$ & $\begin{array}{l}\text { Conv. } \\
0.0164\end{array}$ & $* *$ & $\begin{array}{l}\text { Islamic } \\
0.0592\end{array}$ & $* *$ & $\begin{array}{l}\text { Conv. } \\
0.0381\end{array}$ & $* *$ \\
\hline Pakistan & 0.7432 & $* *$ & 0.0817 & $* *$ & 0.4682 & $* *$ & 0.0574 & $* *$ & 0.0740 & $* * *$ & 0.0021 & $* *$ \\
\hline Saudi Arabia & 0.8342 & $* *$ & 0.0321 & $* *$ & 0.6717 & $* *$ & 0.0293 & $*$ & 0.0648 & $* *$ & 0.0492 & $* * *$ \\
\hline Qatar & 0.6050 & $* *$ & 0.0136 & $* *$ & 0.4257 & $* *$ & 0.0103 & $* *$ & 0.0472 & $* *$ & 0.0215 & $* * *$ \\
\hline Kuwait & 0.7319 & $* *$ & 0.1018 & $* *$ & 0.4503 & $* *$ & 0.0626 & $* *$ & 0.0729 & $* *$ & 0.0330 & $* *$ \\
\hline \multirow[t]{3}{*}{ UAE } & 0.6668 & $* * *$ & 0.1446 & $* *$ & 0.4354 & $* *$ & 0.1001 & $* * *$ & 0.0569 & $* * *$ & 0.0231 & $* *$ \\
\hline & Adjuste & Share & Ratio & & Sortino & $\overline{\text { atio }}$ & & & Jensen' & Alpha & & \\
\hline & Islamic & & Conv. & & Islamic & & Conv. & & Islamic & & Conv. & \\
\hline Malaysia & 0.0174 & $* *$ & -0.3053 & $* *$ & 0.0159 & $* *$ & -0.2297 & $* * *$ & 0.0477 & $* *$ & -0.0295 & $* * *$ \\
\hline Pakistan & 0.0422 & $* *$ & -0.3633 & $* *$ & 0.0300 & $* * *$ & -0.2293 & $* *$ & 0.0507 & $* *$ & -0.0130 & $* *$ \\
\hline Saudi Arabia & 0.0919 & $* *$ & -0.8749 & $* *$ & 0.0759 & $* *$ & -0.5381 & $* *$ & 0.0434 & $* *$ & -0.0548 & $* *$ \\
\hline Qatar & 0.0764 & $* * *$ & -0.0717 & $* * *$ & 0.0629 & $* * *$ & -0.0497 & $* *$ & 0.0353 & $*$ & -0.0310 & $* *$ \\
\hline Kuwait & 0.0816 & $* *$ & -0.0741 & $* *$ & 0.0654 & $*$ & -0.0368 & $* *$ & 0.0401 & $* * *$ & -0.0111 & $* *$ \\
\hline UAE & 0.0991 & $* *$ & -0.0424 & $* *$ & 0.0610 & $* *$ & -0.0298 & $*$ & 0.0432 & $* * *$ & 0.0053 & $* *$ \\
\hline & $\overline{\text { Adjuste }}$ & Share & Ratio & & Sortino & $\overline{\text { atio }}$ & & & $\overline{\text { Jensen' }}$ & Alpha & & \\
\hline & Islamic & & Conv. & & Islamic & & Conv. & & Islamic & & Conv. & \\
\hline Malaysia & 0.0218 & $* *$ & -0.0197 & $* *$ & 0.0141 & $* *$ & -0.0159 & $* *$ & 0.0507 & $* * *$ & -0.0104 & $* *$ \\
\hline Pakistan & 0.0507 & $* *$ & -0.0540 & $* *$ & 0.0375 & $* *$ & -0.0370 & $* *$ & 0.0543 & $* *$ & -0.0034 & $* *$ \\
\hline Saudi Arabia & 0.1016 & $* * *$ & 0.0212 & $* *$ & 0.0975 & $* *$ & 0.0142 & $*$ & 0.0473 & $* * *$ & -0.0022 & $* * *$ \\
\hline Qatar & 0.0994 & $* * *$ & 0.0085 & $* *$ & 0.0791 & $*$ & 0.0063 & $* *$ & 0.0380 & $* *$ & 0.0063 & $* *$ \\
\hline Kuwait & 0.0932 & $* *$ & 0.0694 & $* *$ & 0.0603 & $* *$ & 0.0382 & $* *$ & 0.0426 & $* *$ & 0.0039 & $* *$ \\
\hline UAE & 0.1493 & $* *$ & 0.0428 & $* * *$ & 0.1088 & $* * *$ & 0.0325 & $*$ & 0.0461 & $* *$ & 0.0014 & $* * *$ \\
\hline & Adjuste & Share & Ratio & & Sortino & $\overline{\text { atio }}$ & & & Jensen' & Alpha & & \\
\hline & Islamic & & Conv. & & Islamic & & Conv. & & Islamic & & Conv. & \\
\hline Malaysia & 0.0223 & $* *$ & -0.0177 & $* *$ & 0.0154 & $* *$ & -0.0176 & $* *$ & 0.0548 & $* * *$ & -0.0094 & $* *$ \\
\hline Pakistan & 0.0519 & $* *$ & -0.0486 & $* *$ & 0.0410 & $* *$ & -0.0481 & $* *$ & 0.0587 & $* *$ & -0.0030 & $* *$ \\
\hline Saudi Arabia & 0.1040 & $* * *$ & 0.0191 & $* *$ & 0.1064 & $* *$ & 0.0189 & $*$ & 0.0512 & $* * *$ & -0.0020 & $* * *$ \\
\hline Qatar & 0.1018 & $* * *$ & 0.0076 & $* *$ & 0.0864 & $*$ & 0.0076 & $* *$ & 0.0410 & $* *$ & 0.0057 & $* *$ \\
\hline Kuwait & 0.0954 & $* *$ & -0.0624 & $* *$ & 0.0658 & $* *$ & -0.0618 & $* *$ & 0.0461 & $* *$ & 0.0035 & $* *$ \\
\hline UAE & 0.1528 & $* *$ & 0.0385 & $* * *$ & 0.1187 & $* * *$ & 0.0381 & $*$ & 0.0499 & $* *$ & 0.0013 & $* * *$ \\
\hline & Adjuste & Share & Ratio & & Sortino & $\overline{\text { atio }}$ & & & Jensen' & Alpha & & \\
\hline & Islamic & & Conv. & & Islamic & & Conv. & & Islamic & & Conv. & \\
\hline Malaysia & 0.0279 & $* *$ & 0.0146 & $* *$ & 0.0258 & $* *$ & 0.0015 & $* *$ & 0.0558 & $* * *$ & -0.0084 & $* *$ \\
\hline Pakistan & 0.0625 & $* *$ & 0.0357 & $* *$ & 0.0577 & $* *$ & 0.0036 & $* *$ & 0.0598 & $* *$ & -0.0027 & $* *$ \\
\hline Saudi Arabia & 0.1149 & $* * *$ & 0.0140 & $* *$ & 0.1062 & $* *$ & 0.0014 & $*$ & 0.0521 & $* * *$ & -0.0018 & $* * *$ \\
\hline Qatar & 0.1324 & $* * *$ & 0.0053 & $* *$ & 0.1223 & $*$ & 0.0005 & $* *$ & 0.0418 & $* *$ & -0.0051 & $* *$ \\
\hline Kuwait & 0.1090 & $* *$ & 0.0473 & $* *$ & 0.1007 & $* *$ & 0.0047 & ** & 0.0469 & $* *$ & 0.0032 & $* *$ \\
\hline UAE & 0.2303 & $* *$ & 0.0428 & $* * *$ & 0.2127 & $* * *$ & 0.0043 & * & 0.0508 & $* *$ & 0.0012 & $* * *$ \\
\hline & Adjuste & Share & Ratio & & Sortino & atio & & & Jensen' & Alpha & & \\
\hline & Islamic & & Conv. & & Islamic & & Conv. & & Islamic & & Conv. & \\
\hline Malaysia & 0.0350 & $* *$ & 0.0131 & $* *$ & 0.0332 & $* *$ & 0.0105 & $* *$ & 0.0569 & $* * *$ & -0.0069 & $* *$ \\
\hline Pakistan & 0.0752 & $* *$ & 0.0321 & $* *$ & 0.0715 & $* *$ & 0.0257 & $* *$ & 0.0610 & $* *$ & -0.0022 & $* *$ \\
\hline Saudi Arabia & 0.1271 & $* * *$ & 0.0126 & $* *$ & 0.1207 & $* *$ & 0.0101 & $*$ & 0.0531 & $* * *$ & -0.0015 & $* * *$ \\
\hline Qatar & 0.1723 & $* * *$ & 0.0048 & $* *$ & 0.1637 & $*$ & 0.0038 & $* *$ & 0.0426 & $* *$ & -0.0042 & $* *$ \\
\hline Kuwait & 0.1245 & $* *$ & -0.0425 & $* *$ & 0.1183 & $* *$ & -0.0340 & $* *$ & 0.0478 & $* *$ & 0.0026 & $* *$ \\
\hline UAE & 0.3471 & $* *$ & 0.0428 & $* * *$ & 0.3297 & $* * *$ & 0.0342 & $*$ & 0.0518 & $* *$ & 0.0010 & $* * *$ \\
\hline
\end{tabular}

$* * *$ represents significance at $1 \%, * *$ at $5 \%$ and $*$ at $10 \%$. 


$$
R_{i t}=\left[b_{i, 1} I_{1 t}+b_{i, 2} I_{2 t}+\ldots . .+b_{i, n} I_{n t}\right]+e_{i, t}
$$

$\mathrm{I}_{\mathrm{t}}$ represents the return on style benchmark, $\mathrm{b}_{\mathrm{it}}$ is the percentage of the funds return explained by the style. The model advocates that the variation in fund's return is reflected by the style factor $b_{i, t}$ such that it minimizes $e_{i, t}$. This results in the following:

$$
\operatorname{Min} \sum_{t=1}^{T} e_{i, t}^{2}=\operatorname{Min} \sum_{t=1}^{T}\left(R_{i, t}-\left(b_{i, 1} I_{1 t}+b_{i, 2} I_{2 t}+\ldots .+b_{i, n} I_{n t}\right)\right)^{2}
$$

with the constraints $\sum_{k=1}^{n} b_{i, k}=10 \leq b_{i, k} \leq 1 \mathrm{k}=1,2,3, \ldots \mathrm{n}$.

To analyze the investment styles for Islamic and conventional equity funds, we use size (large, mid, and small), factors (value, growth, and momentum), and five sectoral benchmarks (MSCI). This analysis aims to assess the possible drift in investment preferences during the outbreak. We conduct style analysis for all six stages and not for the entire period.

\subsection{Volatility timing during pandemic}

The volatility timing is important for the robust performance of fund managers. The volatility timing is aimed to optimize the risk by increasing allocations in less volatile asset classes, which results in an improvement in equity funds' performance. The fund managers that can manage volatility scaled investments tend to show better alphas and risk-adjusted metrics. This becomes even more critical during periods of high volatility Hsu and Chen (2017). Moreira and Muir (2019) suggested that funds managers who do not time volatility face a significant risk of losing funds' value. To test for volatility timing, we use the Busse (1999) framework as employed previously by Shen et al. (2019). We specify the functional form of volatility timing as below:

$$
\begin{aligned}
& R_{i t}=\alpha_{i}+\beta_{i} R_{x t}+\gamma_{i m}\left(\sigma_{m t}-\overline{\sigma_{m}}\right) R_{x t}+\varepsilon_{i t} \text { with } R_{x t}=R_{m t}-R_{f t} \\
& R_{x t}=\varphi_{0}+\sum_{p=1}^{n} \varphi_{p} R_{x, t-p}+\varepsilon_{t}-\sum_{p=1}^{q} v_{p} \varepsilon_{t-p} \text { and } \sigma_{m t}^{2}=a_{0}+\sum_{p=1}^{m} a_{p} \varepsilon_{m, t-p}^{2}+\sum_{j=1}^{s} b_{j} \sigma_{m, t-j}^{2}
\end{aligned}
$$

If our sample funds demonstrate volatility timing, the coefficient on volatility timing $\gamma_{\mathrm{im}}$ will be less than zero. We assess both Islamic and conventional funds' volatility timing for the entire period and during the six specified stages of the COVID-19 pandemic.

The data for this study is obtained from multiple sources. Wherever available, NAVs, Benchmark prices, risk-free rates, investment concentration, etc., are extracted from Morningstar, Thomson Reuters Ekon, and Trading Economics. However, for many funds, the information was downloaded from individual websites. Similarly, for some benchmarks, the data was obtained directly from the exchange source.

\section{Results and discussion}

The results on risk-adjusted performance using adjusted Sharpe ratio, Sortino ratio, and Jensen's alpha are presented in Table 4. For the entire period, Islamic equity funds in all six countries demonstrated a positive adjusted Sharpe ratio. In contrast, we report negative adjusted Sharpe ratios for Malaysia, Pakistan, and Saudi Arabia for conventional funds. The equity funds in these countries cannot sustain the COVID-19 pressure and plunged towards negative returns. For traditional funds in Qatar, Kuwait, and UAE, the adjusted Sharpe ratio is positive but still less than their Islamic counterparts. Within Islamic funds, the ones domiciled in Malaysia show a maximum adjusted Sharpe ratio, followed by those in Saudi Arabia. The results are similar for the Sortino ratio, where only the downside risk is considered. Our sample of Islamic funds demonstrates superior downside risk-adjusted performance compared to conventional funds. The Saudi Arabian funds ranked at the top with a Sortino ratio of 0.63 . The least Sortino ratio among this category is for UAE (0.2). Like the adjusted Sharpe ratio, the Sortino ratio for conventional funds in Malaysia, Pakistan, and Saudi Arabia are negative.

The statistics on Jensen's alpha support our findings from adjusted Sharpe and Sortino ratios. For Islamic funds, we report a significant positive alpha across all countries. Contrary to this, conventional funds show a negative alpha for all countries except UAE. This represents that while Islamic funds could dominate the local benchmarks during the outbreak, the conventional funds struggled to do so. The Islamic funds in Pakistan have the maximum alpha (0.06), while those in Saudi Arabia report the least favorable (0.02) in this category. Therefore, for the entire COVID-19 period, we can conclude that the Islamic funds remained resilient during the outbreak than their conventional counterparts.

The results for the six stages are more or less similar. During stage 1, while COVID-19 was in the emerging phase, both Islamic and conventional funds demonstrate positive adjusted Sharpe and Sortino ratios. Furthermore, during this period, Islamic funds dominated the selected traditional funds, which was persistent across all countries. Notably, in stage 1 , Islamic funds in Malaysia have maximum adjusted Sharpe (0.97) and Sortino (0.79) ratios. Among conventional funds, UAE-based funds are ranked at the top of our sample with an adjusted Sharpe ratio of 0.14 and Sortino ratio of 0.10 . Jensen's alpha presents a similar story with positive alphas across all funds. However, we observe higher alphas for Islamic funds vis-à-vis conventional funds. The estimates for stage 1 are mostly significant at $1 \%$ and $5 \%$.

The transition to stage 2 resulted in a performance rout. While the adjusted Sharpe and Sortino ratios are still positive, we observe a comprehensive drop from the level of stage 1 . This is plausible because the intensity of the spread of COVID-19 is resulting in an 
increase in uncertainty and, consequently, reflected in the investment portfolios. We observe a maximum adjusted Sharpe ratio of 0.09 for UAE during this stage, while the Sortino ratio was maximum for Saudi Arabia (0.07). Stage 2 has been highly devastating for the conventional funds that resulted in adverse risk-adjusted performance. The results remained robust for Jensen's alpha with significant positive alphas for all Islamic funds. The traditional funds have negative alphas except for UAE-based funds with a positive alpha of 0.005 for stage 2 . The findings for stage 2 establish that Islamic funds are buoyant and can withstand turbulent market conditions.

In stage 3, we observe a slight improvement in the performance of funds. The Islamic funds show better adjusted Sharpe and Sortino

Table 5

Stochastic dominance (SD1, SD2, SD3 and SD4).

\begin{tabular}{|c|c|c|c|c|}
\hline \multicolumn{5}{|c|}{ Covid19 - Complete Period (Jan 1 - May 31, 2021) } \\
\hline & SD (1) & SD (2) & SD (3) & SD (4) \\
\hline Malaysia & $\mathrm{D}(\mathrm{I}>\mathrm{C})$ & $\mathrm{D}(\mathrm{I}>\mathrm{C})$ & $\mathrm{D}(\mathrm{I}>\mathrm{C})$ & $\mathrm{D}(\mathrm{I}>\mathrm{C})$ \\
\hline Pakistan & $\mathrm{D}(\mathrm{I}>\mathrm{C})$ & $\mathrm{D}(\mathrm{I}>\mathrm{C})$ & $\mathrm{D}(\mathrm{I}>\mathrm{C})$ & $\mathrm{D}(\mathrm{I}>\mathrm{C})$ \\
\hline Saudi Arabia & $\mathrm{D}(\mathrm{I}>\mathrm{C})$ & $\mathrm{D}(\mathrm{I}>\mathrm{C})$ & $\mathrm{D}(\mathrm{I}>\mathrm{C})$ & $\mathrm{D}(\mathrm{I}>\mathrm{C})$ \\
\hline Qatar & $\mathrm{D}(\mathrm{I}>\mathrm{C})$ & $\mathrm{D}(\mathrm{I}>\mathrm{C})$ & $\mathrm{D}(\mathrm{I}>\mathrm{C})$ & $\mathrm{D}(\mathrm{I}>\mathrm{C})$ \\
\hline Kuwait & $\mathrm{D}(\mathrm{I}>\mathrm{C})$ & $\mathrm{D}(\mathrm{I}>\mathrm{C})$ & $\mathrm{D}(\mathrm{I}>\mathrm{C})$ & $\mathrm{D}(\mathrm{I}>\mathrm{C})$ \\
\hline UAE & $\mathrm{D}(\mathrm{I}>\mathrm{C})$ & $\mathrm{D}(\mathrm{I}>\mathrm{C})$ & $\mathrm{D}(\mathrm{I}>\mathrm{C})$ & $\mathrm{D}(\mathrm{I}>\mathrm{C})$ \\
\hline \multicolumn{5}{|c|}{ Stage 1 (Jan 1 - March 19) } \\
\hline Islamic Funds & SD (1) & SD (2) & SD (3) & SD (4) \\
\hline Malaysia & $D(C>I)$ & $D(C>I)$ & $D(C>I)$ & $D(C>I)$ \\
\hline Pakistan & $\mathrm{D}(\mathrm{I}>\mathrm{C})$ & $\mathrm{D}(\mathrm{I}>\mathrm{C})$ & $\mathrm{D}(\mathrm{I}>\mathrm{C})$ & $\mathrm{D}(\mathrm{I}>\mathrm{C})$ \\
\hline Saudi Arabia & $\mathrm{D}(\mathrm{I}>\mathrm{C})$ & $\mathrm{D}(\mathrm{I}>\mathrm{C})$ & $\mathrm{D}(\mathrm{I}>\mathrm{C})$ & $\mathrm{D}(\mathrm{I}>\mathrm{C})$ \\
\hline Qatar & $\mathrm{D}(\mathrm{I}>\mathrm{C})$ & $\mathrm{D}(\mathrm{I}>\mathrm{C})$ & $\mathrm{D}(\mathrm{I}>\mathrm{C})$ & $\mathrm{D}(\mathrm{I}>\mathrm{C})$ \\
\hline Kuwait & $\mathrm{D}(\mathrm{I}>\mathrm{C})$ & $\mathrm{D}(\mathrm{I}>\mathrm{C})$ & $\mathrm{D}(\mathrm{I}>\mathrm{C})$ & $\mathrm{D}(\mathrm{I}>\mathrm{C})$ \\
\hline UAE & $D(C>I)$ & $D(C>I)$ & $D(C>I)$ & $D(C>I)$ \\
\hline \multicolumn{5}{|c|}{ Stage 2 (March 20 - May 11) } \\
\hline Islamic Funds & SD (1) & SD (2) & SD (3) & SD (4) \\
\hline Malaysia & $\mathrm{D}(\mathrm{I}>\mathrm{C})$ & $\mathrm{D}(\mathrm{I}>\mathrm{C})$ & $\mathrm{D}(\mathrm{I}>\mathrm{C})$ & $\mathrm{D}(\mathrm{I}>\mathrm{C})$ \\
\hline Pakistan & $\mathrm{D}(\mathrm{I}>\mathrm{C})$ & $\mathrm{D}(\mathrm{I}>\mathrm{C})$ & $\mathrm{D}(\mathrm{I}>\mathrm{C})$ & $\mathrm{D}(\mathrm{I}>\mathrm{C})$ \\
\hline Saudi Arabia & $\mathrm{D}(\mathrm{I}>\mathrm{C})$ & $\mathrm{D}(\mathrm{I}>\mathrm{C})$ & $\mathrm{D}(\mathrm{I}>\mathrm{C})$ & $\mathrm{D}(\mathrm{I}>\mathrm{C})$ \\
\hline Qatar & $\mathrm{D}(\mathrm{I}>\mathrm{C})$ & $\mathrm{D}(\mathrm{I}>\mathrm{C})$ & $\mathrm{D}(\mathrm{I}>\mathrm{C})$ & $\mathrm{D}(\mathrm{I}>\mathrm{C})$ \\
\hline Kuwait & $\mathrm{D}(\mathrm{I}>\mathrm{C})$ & $\mathrm{D}(\mathrm{I}>\mathrm{C})$ & $\mathrm{D}(\mathrm{I}>\mathrm{C})$ & $\mathrm{D}(\mathrm{I}>\mathrm{C})$ \\
\hline UAE & $\mathrm{D}(\mathrm{I}>\mathrm{C})$ & $\mathrm{D}(\mathrm{I}>\mathrm{C})$ & $\mathrm{D}(\mathrm{I}>\mathrm{C})$ & $\mathrm{D}(\mathrm{I}>\mathrm{C})$ \\
\hline \multicolumn{5}{|c|}{ 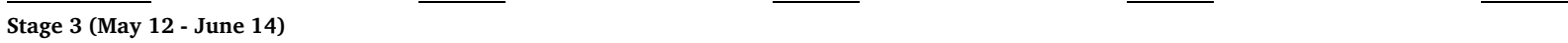 } \\
\hline & SD (1) & SD (2) & SD (3) & SD (4) \\
\hline Malaysia & $\mathrm{D}(\mathrm{I}>\mathrm{C})$ & $\mathrm{D}(\mathrm{I}>\mathrm{C})$ & $\mathrm{D}(\mathrm{I}>\mathrm{C})$ & $\mathrm{D}(\mathrm{I}>\mathrm{C})$ \\
\hline Pakistan & $\mathrm{D}(\mathrm{I}>\mathrm{C})$ & $\mathrm{D}(\mathrm{I}>\mathrm{C})$ & $\mathrm{D}(\mathrm{I}>\mathrm{C})$ & $\mathrm{D}(\mathrm{I}>\mathrm{C})$ \\
\hline Saudi Arabia & $\mathrm{D}(\mathrm{I}>\mathrm{C})$ & $\mathrm{D}(\mathrm{I}>\mathrm{C})$ & $\mathrm{D}(\mathrm{I}>\mathrm{C})$ & $\mathrm{D}(\mathrm{I}>\mathrm{C})$ \\
\hline Qatar & $\mathrm{D}(\mathrm{I}>\mathrm{C})$ & $\mathrm{D}(\mathrm{I}>\mathrm{C})$ & $\mathrm{D}(\mathrm{I}>\mathrm{C})$ & $\mathrm{D}(\mathrm{I}>\mathrm{C})$ \\
\hline Kuwait & $\mathrm{D}(\mathrm{I}>\mathrm{C})$ & $\mathrm{D}(\mathrm{I}>\mathrm{C})$ & $\mathrm{D}(\mathrm{I}>\mathrm{C})$ & $\mathrm{D}(\mathrm{I}>\mathrm{C})$ \\
\hline UAE & $\underline{D(I>C)}$ & $\underline{D(I>C)}$ & $\underline{D(I>C)}$ & $\mathrm{D}(\mathrm{I}>\mathrm{C})$ \\
\hline \multicolumn{5}{|c|}{ 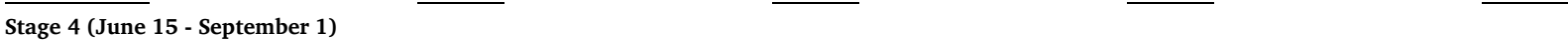 } \\
\hline & SD (1) & SD (2) & SD (3) & SD (4) \\
\hline Malaysia & $\mathrm{D}(\mathrm{I}>\mathrm{C})$ & $\mathrm{D}(\mathrm{I}>\mathrm{C})$ & $\mathrm{D}(\mathrm{I}>\mathrm{C})$ & $\mathrm{D}(\mathrm{I}>\mathrm{C})$ \\
\hline Pakistan & $\mathrm{D}(\mathrm{I}>\mathrm{C})$ & $\mathrm{D}(\mathrm{I}>\mathrm{C})$ & $\mathrm{D}(\mathrm{I}>\mathrm{C})$ & $\mathrm{D}(\mathrm{I}>\mathrm{C})$ \\
\hline Saudi Arabia & $\mathrm{D}(\mathrm{I}>\mathrm{C})$ & $\mathrm{D}(\mathrm{I}>\mathrm{C})$ & $\mathrm{D}(\mathrm{I}>\mathrm{C})$ & $\mathrm{D}(\mathrm{I}>\mathrm{C})$ \\
\hline Qatar & $\mathrm{D}(\mathrm{I}>\mathrm{C})$ & $\mathrm{D}(\mathrm{I}>\mathrm{C})$ & $\mathrm{D}(\mathrm{I}>\mathrm{C})$ & $\mathrm{D}(\mathrm{I}>\mathrm{C})$ \\
\hline Kuwait & $\mathrm{D}(\mathrm{I}>\mathrm{C})$ & $\mathrm{D}(\mathrm{I}>\mathrm{C})$ & $\mathrm{D}(\mathrm{I}>\mathrm{C})$ & $\mathrm{D}(\mathrm{I}>\mathrm{C})$ \\
\hline UAE & $\mathrm{D}(\mathrm{I}>\mathrm{C})$ & $\mathrm{D}(\mathrm{I}>\mathrm{C})$ & $\mathrm{D}(\mathrm{I}>\mathrm{C})$ & $\mathrm{D}(\mathrm{I}>\mathrm{C})$ \\
\hline \multicolumn{5}{|c|}{ Stage 5 (September 2 - December 31) } \\
\hline & SD (1) & SD (2) & SD (3) & SD (4) \\
\hline Malaysia & $\mathrm{D}(\mathrm{I}>\mathrm{C})$ & $\mathrm{D}(\mathrm{I}>\mathrm{C})$ & $\mathrm{D}(\mathrm{I}>\mathrm{C})$ & $\mathrm{D}(\mathrm{I}>\mathrm{C})$ \\
\hline Pakistan & $\mathrm{D}(\mathrm{I}>\mathrm{C})$ & $\mathrm{D}(\mathrm{I}>\mathrm{C})$ & $\mathrm{D}(\mathrm{I}>\mathrm{C})$ & $\mathrm{D}(\mathrm{I}>\mathrm{C})$ \\
\hline Saudi Arabia & $\mathrm{D}(\mathrm{I}>\mathrm{C})$ & $\mathrm{D}(\mathrm{I}>\mathrm{C})$ & $\mathrm{D}(\mathrm{I}>\mathrm{C})$ & $\mathrm{D}(\mathrm{I}>\mathrm{C})$ \\
\hline Qatar & $D(C>I)$ & $D(C>I)$ & $D(C>I)$ & $D(C>I)$ \\
\hline Kuwait & $\mathrm{D}(\mathrm{I}>\mathrm{C})$ & $\mathrm{D}(\mathrm{I}>\mathrm{C})$ & $\mathrm{D}(\mathrm{I}>\mathrm{C})$ & $\mathrm{D}(\mathrm{I}>\mathrm{C})$ \\
\hline UAE & $\mathrm{D}(\mathrm{I}>\mathrm{C})$ & $\mathrm{D}(\mathrm{I}>\mathrm{C})$ & $\mathrm{D}(\mathrm{I}>\mathrm{C})$ & $\mathrm{D}(\mathrm{I}>\mathrm{C})$ \\
\hline \multicolumn{5}{|c|}{ 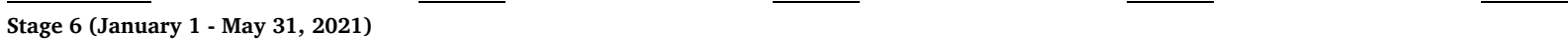 } \\
\hline & SD (1) & SD (2) & SD (3) & SD (4) \\
\hline Malaysia & $\mathrm{D}(\mathrm{I}>\mathrm{C})$ & $\mathrm{D}(\mathrm{I}>\mathrm{C})$ & $\mathrm{D}(\mathrm{I}>\mathrm{C})$ & $\mathrm{D}(\mathrm{I}>\mathrm{C})$ \\
\hline Pakistan & $\mathrm{D}(\mathrm{I}>\mathrm{C})$ & $\mathrm{D}(\mathrm{I}>\mathrm{C})$ & $\mathrm{D}(\mathrm{I}>\mathrm{C})$ & $\mathrm{D}(\mathrm{I}>\mathrm{C})$ \\
\hline Saudi Arabia & $\mathrm{D}(\mathrm{I}>\mathrm{C})$ & $\mathrm{D}(\mathrm{I}>\mathrm{C})$ & $\mathrm{D}(\mathrm{I}>\mathrm{C})$ & $\mathrm{D}(\mathrm{I}>\mathrm{C})$ \\
\hline Qatar & $\mathrm{D}(\mathrm{C}>\mathrm{I})$ & $\mathrm{D}(\mathrm{C}>\mathrm{I})$ & $\mathrm{D}(\mathrm{C}>\mathrm{I})$ & $\mathrm{D}(\mathrm{C}>\mathrm{I})$ \\
\hline Kuwait & $\mathrm{D}(\mathrm{I}>\mathrm{C})$ & $\mathrm{D}(\mathrm{I}>\mathrm{C})$ & $\mathrm{D}(\mathrm{I}>\mathrm{C})$ & $\mathrm{D}(\mathrm{I}>\mathrm{C})$ \\
\hline UAE & $\mathrm{D}(\mathrm{I}>\mathrm{C})$ & $\mathrm{D}(\mathrm{I}>\mathrm{C})$ & $\mathrm{D}(\mathrm{I}>\mathrm{C})$ & $\mathrm{D}(\mathrm{I}>\mathrm{C})$ \\
\hline
\end{tabular}

$\mathrm{I}=$ Islamic Funds, $\mathrm{C}=$ Conventional Funds. 
Table 6a

Cumulative abnormal returns (Islamic and conventional funds).

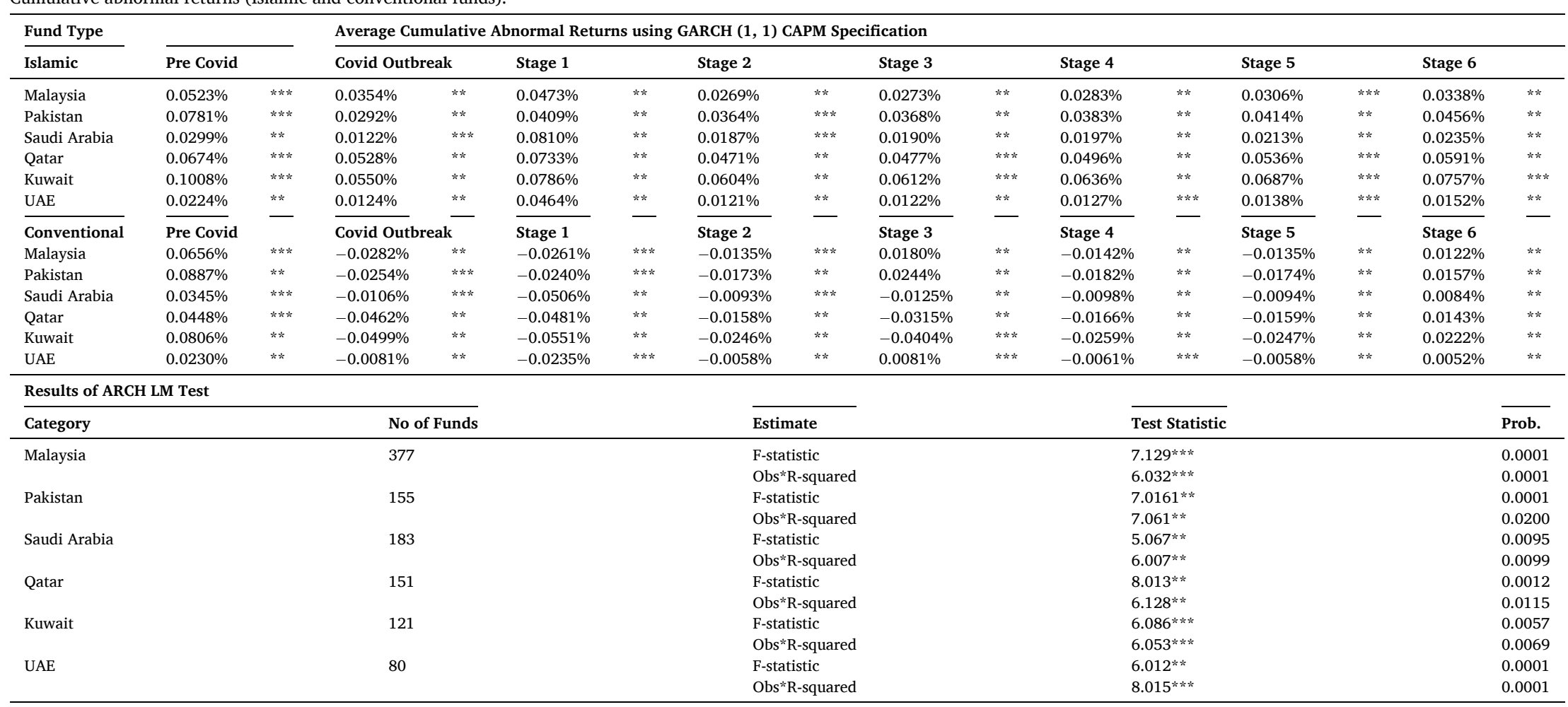

***represent significance at $1 \%, * *$ at $5 \%$ and $*$ at $10 \%$. 
Table 6b

Coefficients and $t$ Stats of Oil Dependence Dummy.

\begin{tabular}{|c|c|c|c|c|c|c|c|c|c|c|c|c|c|c|c|c|c|}
\hline \multirow{2}{*}{$\begin{array}{l}\text { Fund Type } \\
\text { Islamic } \\
\end{array}$} & \multirow{2}{*}{$\begin{array}{l}\text { Oil Rent to } \\
\text { GDP\% } \\
\end{array}$} & \multicolumn{2}{|l|}{ Pre Covid } & \multicolumn{2}{|c|}{ Covid Outbreak } & \multicolumn{2}{|l|}{ Stage 1} & \multicolumn{2}{|l|}{ Stage 2} & \multicolumn{2}{|l|}{ Stage 3} & \multicolumn{2}{|l|}{ Stage 4} & \multicolumn{2}{|l|}{ Stage 5} & \multicolumn{2}{|l|}{ Stage 6} \\
\hline & & Coefficient & t stats & Coefficient & t stats & Coefficient & t stats & Coefficient & $\mathrm{t}$ stats & Coefficient & $\mathrm{t}$ stats & Coefficient & t stats & Coefficient & $\mathrm{t}$ stats & Coefficient & $\mathrm{t}$ stats \\
\hline Malaysia & $4.58 \%$ & 0.6480 & 1.4010 & 0.9213 & 1.0259 & 0.4188 & 0.7784 & 0.4604 & 0.0147 & 0.2723 & 0.7819 & 0.3099 & 0.0260 & 0.8518 & 0.8024 & 0.6630 & 0.9966 \\
\hline Pakistan & $0.70 \%$ & 0.8842 & 0.5988 & 0.9319 & 0.9170 & 0.0214 & 0.8186 & 0.2106 & 0.8563 & 0.8138 & 0.8875 & 0.5338 & 0.4731 & 0.8462 & 0.5987 & 0.0678 & 0.5718 \\
\hline Saudi Arabia & $39.06 \%$ & 0.6924 & 0.8387 & 0.3874 & 1.1727 & 0.5125 & 0.0340 & 0.9997 & 0.9290 & 0.3775 & 0.9263 & 0.2960 & 0.0507 & 0.3895 & 0.4753 & 0.8712 & 0.9076 \\
\hline Qatar & $27.30 \%$ & 0.6962 & 1.3718 & 0.4455 & 0.0946 & 0.6100 & 0.7275 & 0.3771 & 0.3015 & 0.7420 & 0.3349 & 0.9266 & 1.1329 & 0.8943 & 0.8522 & 0.6690 & 0.6511 \\
\hline Kuwait & $47.33 \%$ & 0.2638 & 1.3866 & 0.6389 & 0.0313 & 0.5979 & 0.8828 & 0.4371 & 0.2341 & 0.1050 & 0.0925 & 0.6006 & 0.3364 & 0.2704 & 0.3965 & 0.8694 & 0.7882 \\
\hline UAE & $20.47 \%$ & 0.7472 & 0.1572 & 0.3483 & 0.0382 & 0.6966 & 0.9221 & 0.6889 & 0.5779 & 0.6458 & 1.2032 & 0.1148 & 0.8563 & 0.0477 & 0.0068 & 0.1516 & 0.1194 \\
\hline Conventional & & & & & & & & & & & & & & & & & \\
\hline Malaysia & $4.58 \%$ & 0.3168 & 0.6561 & 0.0878 & 0.9443 & 0.2563 & 0.5045 & 0.8056 & 0.6321 & 0.4774 & 0.0550 & 0.6642 & 0.0753 & 0.9406 & 0.1241 & 0.9097 & 0.2838 \\
\hline Pakistan & $0.70 \%$ & 0.5006 & 0.5097 & 0.9037 & 1.2241 & 0.4129 & 0.0530 & 0.2117 & 0.0882 & 0.9048 & 0.0842 & 0.4800 & 0.6574 & 0.5576 & 0.6399 & 0.6143 & 0.6151 \\
\hline Saudi Arabia & $39.06 \%$ & 0.7508 & 1.0898 & 0.1328 & 0.0571 & 0.6947 & 0.0764 & 0.7683 & 0.2343 & 0.6289 & 0.7025 & 0.3842 & 0.8886 & 0.1264 & 0.0080 & 0.7211 & 0.5127 \\
\hline Qatar & $27.30 \%$ & 0.3754 & 1.2001 & 0.1635 & 0.9665 & 0.7421 & 0.9033 & 0.9886 & 1.0184 & 0.3992 & 0.9191 & 0.4015 & 1.2589 & 0.8011 & 0.9529 & 0.0956 & 0.2849 \\
\hline Kuwait & $47.33 \%$ & 0.4373 & 0.8678 & 0.2129 & 0.1362 & 0.6082 & 1.0273 & 0.9111 & 0.3195 & 0.6054 & 0.9478 & 0.6760 & 0.7539 & 0.2642 & 0.0849 & 0.4924 & 0.4710 \\
\hline UAE & $20.47 \%$ & 0.9388 & 0.4933 & 0.8829 & 0.8333 & 0.0785 & 0.4689 & 0.1251 & 0.0315 & 0.5444 & 0.0816 & 0.1206 & 0.0300 & 0.0702 & 0.3060 & 0.0370 & 0.5838 \\
\hline
\end{tabular}


Table 7

Style analysis equity funds - stage 1 (January 1st - March 19).

\begin{tabular}{|c|c|c|c|c|c|c|c|c|c|c|c|c|}
\hline & \multicolumn{2}{|c|}{ Malaysia } & \multicolumn{2}{|c|}{ Pakistan } & \multicolumn{2}{|c|}{ Saudi Arabia } & \multicolumn{2}{|l|}{ Qatar } & \multicolumn{2}{|l|}{ Kuwait } & \multicolumn{2}{|l|}{ UAE } \\
\hline & Islamic & Conv. & Islamic & Conv. & Islamic & Conv. & Islamic & Conv. & Islamic & Conv. & Islamic & Conv. \\
\hline \multicolumn{13}{|l|}{ Size } \\
\hline Large & $45.10 \%$ & $23.15 \%$ & $38.45 \%$ & $25.15 \%$ & $35.13 \%$ & $20.15 \%$ & $31.59 \%$ & $19.55 \%$ & $40.13 \%$ & $25.68 \%$ & $43.12 \%$ & $21.50 \%$ \\
\hline Mid & $21.20 \%$ & $15.55 \%$ & $35.66 \%$ & $31.23 \%$ & $31.25 \%$ & $25.45 \%$ & $42.56 \%$ & $31.55 \%$ & $31.25 \%$ & $35.55 \%$ & $21.25 \%$ & $23.60 \%$ \\
\hline Small & $33.70 \%$ & $61.30 \%$ & $25.89 \%$ & $43.62 \%$ & $33.63 \%$ & $54.41 \%$ & $25.85 \%$ & $48.90 \%$ & $28.63 \%$ & $38.77 \%$ & $35.63 \%$ & $54.90 \%$ \\
\hline $\mathrm{R} 2$ & 0.931 & 0.851 & 0.864 & 0.903 & 0.912 & 0.876 & 0.860 & 0.893 & 0.917 & 0.925 & 0.951 & 0.969 \\
\hline Tracking Error & $0.55 \%$ & $0.68 \%$ & $0.96 \%$ & $0.67 \%$ & $0.93 \%$ & $0.72 \%$ & $0.97 \%$ & $0.38 \%$ & $0.65 \%$ & $0.89 \%$ & $0.63 \%$ & $0.96 \%$ \\
\hline \multicolumn{13}{|l|}{ Factor Strategies } \\
\hline Growth & $35.50 \%$ & $55.30 \%$ & $31.55 \%$ & $55.15 \%$ & $22.51 \%$ & $52.20 \%$ & $62.25 \%$ & $23.15 \%$ & $14.15 \%$ & $44.68 \%$ & $21.05 \%$ & $50.12 \%$ \\
\hline Value & $45.10 \%$ & $25.50 \%$ & $52.20 \%$ & $22.20 \%$ & $33.10 \%$ & $15.57 \%$ & $22.50 \%$ & $24.45 \%$ & $55.61 \%$ & $23.51 \%$ & $29.55 \%$ & $15.56 \%$ \\
\hline Momentum & $19.40 \%$ & $19.20 \%$ & $16.25 \%$ & $22.65 \%$ & $44.39 \%$ & $32.23 \%$ & $15.25 \%$ & $52.40 \%$ & $30.24 \%$ & $31.81 \%$ & $49.40 \%$ & $34.32 \%$ \\
\hline $\mathrm{R} 2$ & 0.923 & 0.892 & 0.881 & 0.943 & 0.873 & 0.921 & 0.867 & 0.948 & 0.958 & 0.870 & 0.923 & 0.918 \\
\hline Tracking Error & $0.90 \%$ & $0.46 \%$ & $0.87 \%$ & $0.91 \%$ & $0.40 \%$ & $0.44 \%$ & $0.77 \%$ & $0.79 \%$ & $0.60 \%$ & $0.79 \%$ & $0.52 \%$ & $0.59 \%$ \\
\hline \multicolumn{13}{|l|}{ Sector } \\
\hline Manufacturing & $40.13 \%$ & $42.56 \%$ & $30.25 \%$ & $46.12 \%$ & $29.15 \%$ & $31.03 \%$ & $25.23 \%$ & $31.02 \%$ & $21.51 \%$ & $32.05 \%$ & $29.15 \%$ & $41.02 \%$ \\
\hline Utilities & $12.51 \%$ & $10.25 \%$ & $10.25 \%$ & $9.11 \%$ & $20.10 \%$ & $23.15 \%$ & $19.25 \%$ & $18.25 \%$ & $20.12 \%$ & $21.05 \%$ & $18.15 \%$ & $15.12 \%$ \\
\hline Mining, Construction and Chemicals & $15.50 \%$ & $23.31 \%$ & $25.12 \%$ & $13.51 \%$ & $28.91 \%$ & $20.15 \%$ & $21.35 \%$ & $10.21 \%$ & $21.55 \%$ & $12.52 \%$ & $21.54 \%$ & $12.54 \%$ \\
\hline Wholesale and Retail & $21.25 \%$ & $10.23 \%$ & $20.14 \%$ & $12.57 \%$ & $10.25 \%$ & $15.32 \%$ & $20.15 \%$ & $21.26 \%$ & $19.15 \%$ & $21.25 \%$ & $25.45 \%$ & $23.51 \%$ \\
\hline Services & $10.61 \%$ & $13.66 \%$ & $14.24 \%$ & $18.69 \%$ & $11.60 \%$ & $10.36 \%$ & $14.02 \%$ & $19.26 \%$ & $17.67 \%$ & $13.13 \%$ & $5.71 \%$ & $7.81 \%$ \\
\hline R2 & 0.924 & 0.935 & 0.862 & 0.907 & 0.911 & 0.964 & 0.902 & 0.935 & 0.892 & 0.902 & 0.855 & 0.865 \\
\hline Tracking Error & $0.31 \%$ & $0.35 \%$ & $0.40 \%$ & $0.52 \%$ & $0.91 \%$ & $0.98 \%$ & $0.59 \%$ & $0.63 \%$ & $0.79 \%$ & $0.64 \%$ & $0.44 \%$ & $0.95 \%$ \\
\hline
\end{tabular}


Table 8

Style analysis equity funds - stage 2 (March 20 - May 11).

\begin{tabular}{|c|c|c|c|c|c|c|c|c|c|c|c|c|}
\hline & \multicolumn{2}{|c|}{ Malaysia } & \multicolumn{2}{|c|}{ Pakistan } & \multicolumn{2}{|c|}{ Saudi Arabia } & \multicolumn{2}{|l|}{ Qatar } & \multicolumn{2}{|l|}{ Kuwait } & \multicolumn{2}{|l|}{ UAE } \\
\hline & Islamic & Conv. & Islamic & Conv. & Islamic & Conv. & Islamic & Conv. & Islamic & Conv. & Islamic & Conv. \\
\hline \multicolumn{13}{|l|}{ Size } \\
\hline Large & $54.23 \%$ & $24.35 \%$ & $48.15 \%$ & $25.48 \%$ & $45.43 \%$ & $20.78 \%$ & $41.11 \%$ & $21.35 \%$ & $58.28 \%$ & $26.48 \%$ & $53.28 \%$ & $21.77 \%$ \\
\hline Mid & $31.02 \%$ & $23.15 \%$ & $31.45 \%$ & $32.25 \%$ & $35.22 \%$ & $25.45 \%$ & $39.15 \%$ & $32.15 \%$ & $31.45 \%$ & $37.25 \%$ & $25.40 \%$ & $31.55 \%$ \\
\hline Small & $14.75 \%$ & $52.50 \%$ & $20.40 \%$ & $42.28 \%$ & $19.35 \%$ & $53.77 \%$ & $19.74 \%$ & $46.50 \%$ & $10.27 \%$ & $36.27 \%$ & $21.32 \%$ & $46.68 \%$ \\
\hline $\mathrm{R} 2$ & 0.871 & 0.923 & 0.866 & 0.932 & 0.853 & 0.875 & 0.925 & 0.874 & 0.896 & 0.832 & 0.937 & 0.877 \\
\hline $\begin{array}{l}\text { Tracking Error } \\
\text { Factor Strategies }\end{array}$ & $0.85 \%$ & $0.55 \%$ & $0.70 \%$ & $0.78 \%$ & $0.97 \%$ & $0.70 \%$ & $0.52 \%$ & $0.88 \%$ & $0.97 \%$ & $0.81 \%$ & $0.85 \%$ & $0.88 \%$ \\
\hline Growth & $25.05 \%$ & $55.98 \%$ & $17.24 \%$ & $55.84 \%$ & $18.29 \%$ & $52.26 \%$ & $40.12 \%$ & $22.04 \%$ & $11.32 \%$ & $43.90 \%$ & $21.23 \%$ & $51.75 \%$ \\
\hline Value & $55.01 \%$ & $25.81 \%$ & $67.93 \%$ & $22.48 \%$ & $45.08 \%$ & $15.59 \%$ & $31.53 \%$ & $23.28 \%$ & $73.47 \%$ & $23.10 \%$ & $42.85 \%$ & $16.07 \%$ \\
\hline Momentum & $19.94 \%$ & $18.20 \%$ & $14.84 \%$ & $21.68 \%$ & $36.63 \%$ & $32.15 \%$ & $28.35 \%$ & $54.68 \%$ & $15.21 \%$ & $33.00 \%$ & $35.92 \%$ & $32.18 \%$ \\
\hline $\mathrm{R} 2$ & 0.916 & 0.912 & 0.877 & 0.916 & 0.949 & 0.951 & 0.951 & 0.839 & 0.905 & 0.913 & 0.935 & 0.903 \\
\hline $\begin{array}{l}\text { Tracking Error } \\
\text { Sector }\end{array}$ & \multicolumn{11}{|c|}{ Sector } & $0.44 \%$ \\
\hline Manufacturing & $30.19 \%$ & $37.97 \%$ & $22.15 \%$ & $43.92 \%$ & $21.93 \%$ & $31.26 \%$ & $13.94 \%$ & $26.44 \%$ & $14.03 \%$ & $32.29 \%$ & $24.85 \%$ & $30.86 \%$ \\
\hline Utilities & $13.83 \%$ & $10.80 \%$ & $11.82 \%$ & $9.94 \%$ & $22.22 \%$ & $23.96 \%$ & $22.15 \%$ & $20.17 \%$ & $24.05 \%$ & $22.85 \%$ & $20.42 \%$ & $16.71 \%$ \\
\hline Mining, Construction and Chemicals & $11.66 \%$ & $19.87 \%$ & $18.90 \%$ & $12.88 \%$ & $21.75 \%$ & $17.24 \%$ & $16.06 \%$ & $9.72 \%$ & $18.37 \%$ & $8.17 \%$ & $11.90 \%$ & $9.43 \%$ \\
\hline Wholesale and Retail & $32.56 \%$ & $12.81 \%$ & $31.66 \%$ & $15.49 \%$ & $15.71 \%$ & $20.41 \%$ & $32.89 \%$ & $28.11 \%$ & $31.26 \%$ & $28.31 \%$ & $39.25 \%$ & $36.02 \%$ \\
\hline Services & $11.76 \%$ & $18.55 \%$ & $15.46 \%$ & $17.77 \%$ & $18.40 \%$ & $7.13 \%$ & $14.97 \%$ & $15.56 \%$ & $12.29 \%$ & $8.39 \%$ & $3.58 \%$ & $6.97 \%$ \\
\hline R2 & 0.864 & 0.955 & 0.960 & 0.898 & 0.952 & 0.905 & 0.894 & 0.947 & 0.891 & 0.931 & 0.877 & 0.875 \\
\hline Tracking Error & $0.78 \%$ & $0.77 \%$ & $0.90 \%$ & $0.77 \%$ & $0.79 \%$ & $0.88 \%$ & $0.53 \%$ & $0.94 \%$ & $0.71 \%$ & $0.98 \%$ & $0.71 \%$ & $0.78 \%$ \\
\hline
\end{tabular}


Table 9

Style analysis - stage 3 (May 12 - June 14).

\begin{tabular}{|c|c|c|c|c|c|c|c|c|c|c|c|c|}
\hline & \multicolumn{2}{|c|}{ Malaysia } & \multicolumn{2}{|c|}{ Pakistan } & \multicolumn{2}{|c|}{ Saudi Arabia } & \multicolumn{2}{|l|}{ Qatar } & \multicolumn{2}{|l|}{ Kuwait } & \multicolumn{2}{|l|}{ UAE } \\
\hline & Islamic & Conv. & Islamic & Conv. & Islamic & Conv. & Islamic & Conv. & Islamic & Conv. & Islamic & Conv. \\
\hline \multicolumn{13}{|l|}{ Size } \\
\hline Large & $67.05 \%$ & $25.24 \%$ & $51.31 \%$ & $26.56 \%$ & $60.45 \%$ & $22.14 \%$ & $58.11 \%$ & $21.40 \%$ & $72.89 \%$ & $29.75 \%$ & $71.21 \%$ & $23.78 \%$ \\
\hline Mid & $18.24 \%$ & $31.25 \%$ & $25.66 \%$ & $43.56 \%$ & $26.57 \%$ & $35.58 \%$ & $22.34 \%$ & $31.55 \%$ & $12.36 \%$ & $35.66 \%$ & $15.33 \%$ & $41.33 \%$ \\
\hline Small & $14.71 \%$ & $43.51 \%$ & $23.03 \%$ & $29.88 \%$ & $12.98 \%$ & $42.28 \%$ & $19.55 \%$ & $47.05 \%$ & $14.75 \%$ & $34.59 \%$ & $13.47 \%$ & $34.90 \%$ \\
\hline $\mathrm{R} 2$ & 0.903 & 0.851 & 0.857 & 0.926 & 0.919 & 0.896 & 0.908 & 0.872 & 0.946 & 0.873 & 0.912 & 0.865 \\
\hline $\begin{array}{l}\text { Tracking Error } \\
\text { Factor Strategies }\end{array}$ & \multicolumn{11}{|c|}{ Factor Strategies } & $0.63 \%$ \\
\hline Growth & $19.67 \%$ & $53.32 \%$ & $13.88 \%$ & $56.26 \%$ & $11.93 \%$ & $46.39 \%$ & $22.29 \%$ & $23.03 \%$ & $10.25 \%$ & $44.25 \%$ & $15.97 \%$ & $49.29 \%$ \\
\hline Value & $63.35 \%$ & $23.33 \%$ & $67.13 \%$ & $24.56 \%$ & $59.02 \%$ & $15.52 \%$ & $44.17 \%$ & $31.23 \%$ & $78.38 \%$ & $20.13 \%$ & $48.75 \%$ & $14.55 \%$ \\
\hline Momentum & $16.98 \%$ & $23.35 \%$ & $18.99 \%$ & $19.18 \%$ & $29.05 \%$ & $38.09 \%$ & $33.54 \%$ & $45.74 \%$ & $11.37 \%$ & $35.62 \%$ & $35.28 \%$ & $36.16 \%$ \\
\hline $\mathrm{R} 2$ & 0.873 & 0.957 & 0.851 & 0.941 & 0.862 & 0.948 & 0.861 & 0.854 & 0.879 & 0.883 & 0.904 & 0.896 \\
\hline $\begin{array}{l}\text { Tracking Error } \\
\text { Sector }\end{array}$ & $0.53 \%$ & $0.11 \%$ & $0.84 \%$ & $0.81 \%$ & $0.15 \%$ & $0.46 \%$ & $0.26 \%$ & $0.14 \%$ & $0.30 \%$ & $0.73 \%$ & $0.76 \%$ & $0.89 \%$ \\
\hline Manufacturing & $26.29 \%$ & $36.15 \%$ & $18.16 \%$ & $40.39 \%$ & $18.19 \%$ & $28.95 \%$ & $10.58 \%$ & $22.11 \%$ & $12.56 \%$ & $25.65 \%$ & $20.12 \%$ & $25.48 \%$ \\
\hline Utilities & $15.83 \%$ & $12.12 \%$ & $12.82 \%$ & $12.51 \%$ & $23.16 \%$ & $20.97 \%$ & $23.99 \%$ & $21.64 \%$ & $24.89 \%$ & $23.15 \%$ & $21.12 \%$ & $15.38 \%$ \\
\hline Mining, Construction and Chemicals & $10.66 \%$ & $19.86 \%$ & $15.13 \%$ & $10.88 \%$ & $19.15 \%$ & $16.15 \%$ & $14.56 \%$ & $8.47 \%$ & $15.24 \%$ & $7.72 \%$ & $8.91 \%$ & $6.35 \%$ \\
\hline Wholesale and Retail & $35.56 \%$ & $16.45 \%$ & $39.16 \%$ & $18.84 \%$ & $20.26 \%$ & $25.87 \%$ & $38.15 \%$ & $35.21 \%$ & $35.36 \%$ & $35.73 \%$ & $45.73 \%$ & $44.12 \%$ \\
\hline Services & $11.66 \%$ & $15.42 \%$ & $14.73 \%$ & $17.38 \%$ & $19.24 \%$ & $8.06 \%$ & $12.72 \%$ & $12.57 \%$ & $11.95 \%$ & $7.75 \%$ & $4.12 \%$ & $8.67 \%$ \\
\hline R2 & 0.934 & 0.895 & 0.899 & 0.964 & 0.905 & 0.894 & 0.873 & 0.892 & 0.916 & 0.893 & 0.951 & 0.941 \\
\hline Tracking Error & $0.45 \%$ & $0.49 \%$ & $0.36 \%$ & $0.86 \%$ & $0.79 \%$ & $0.85 \%$ & $0.65 \%$ & $0.79 \%$ & $0.49 \%$ & $0.80 \%$ & $0.51 \%$ & $0.98 \%$ \\
\hline
\end{tabular}


Table 10

Style analysis - stage 4 (June 15 - September 1).

\begin{tabular}{|c|c|c|c|c|c|c|c|c|c|c|c|c|}
\hline & \multicolumn{2}{|c|}{ Malaysia } & \multicolumn{2}{|c|}{ Pakistan } & \multicolumn{2}{|c|}{ Saudi Arabia } & \multicolumn{2}{|l|}{ Qatar } & \multicolumn{2}{|l|}{ Kuwait } & \multicolumn{2}{|l|}{ UAE } \\
\hline & Islamic & Conv. & Islamic & Conv. & Islamic & Conv. & Islamic & Conv. & Islamic & Conv. & Islamic & Conv. \\
\hline \multicolumn{13}{|l|}{ Size } \\
\hline Large & $82.92 \%$ & $31.22 \%$ & $63.45 \%$ & $32.84 \%$ & $74.74 \%$ & $27.38 \%$ & $71.86 \%$ & $26.46 \%$ & $90.13 \%$ & $36.79 \%$ & $88.05 \%$ & $29.40 \%$ \\
\hline Mid & $15.24 \%$ & $33.15 \%$ & $23.35 \%$ & $39.64 \%$ & $24.18 \%$ & $32.38 \%$ & $20.33 \%$ & $28.71 \%$ & $11.25 \%$ & $32.45 \%$ & $13.95 \%$ & $37.61 \%$ \\
\hline Small & $1.84 \%$ & $35.63 \%$ & $13.20 \%$ & $27.52 \%$ & $1.08 \%$ & $40.24 \%$ & $7.82 \%$ & $44.83 \%$ & $-1.38 \%$ & $30.77 \%$ & $-2.00 \%$ & $32.99 \%$ \\
\hline $\mathrm{R} 2$ & 0.902 & 0.871 & 0.957 & 0.902 & 0.929 & 0.880 & 0.902 & 0.820 & 0.902 & 0.860 & 0.901 & 0.880 \\
\hline $\begin{array}{l}\text { Tracking Error } \\
\text { Factor Strategies }\end{array}$ & & $0.57 \%$ \\
\hline Growth & $19.28 \%$ & $52.25 \%$ & $13.60 \%$ & $55.14 \%$ & $11.69 \%$ & $45.46 \%$ & $21.84 \%$ & $22.57 \%$ & $10.04 \%$ & $43.37 \%$ & $15.65 \%$ & $48.30 \%$ \\
\hline Value & $62.08 \%$ & $22.86 \%$ & $65.79 \%$ & $24.06 \%$ & $57.84 \%$ & $15.21 \%$ & $43.29 \%$ & $30.60 \%$ & $76.81 \%$ & $19.72 \%$ & $47.78 \%$ & $14.26 \%$ \\
\hline Momentum & $18.64 \%$ & $24.89 \%$ & $20.61 \%$ & $20.80 \%$ & $30.47 \%$ & $39.33 \%$ & $34.87 \%$ & $46.83 \%$ & $13.15 \%$ & $36.91 \%$ & $36.57 \%$ & $37.44 \%$ \\
\hline $\mathrm{R} 2$ & 0.8294 & 0.9092 & 0.8085 & 0.8937 & 0.819 & 0.9006 & 0.818 & 0.8113 & 0.8351 & 0.8389 & 0.8588 & 0.8512 \\
\hline Tracking Error & 0.005 & 0.001 & 0.008 & 0.0077 & 0.0014 & 0.0044 & 0.0025 & 0.0013 & 0.0029 & 0.0069 & 0.0072 & 0.0085 \\
\hline \multicolumn{13}{|l|}{ Sector } \\
\hline Manufacturing & $24.28 \%$ & $33.39 \%$ & $16.77 \%$ & $37.31 \%$ & $16.80 \%$ & $26.74 \%$ & $9.77 \%$ & $20.42 \%$ & $11.60 \%$ & $23.69 \%$ & $18.58 \%$ & $23.53 \%$ \\
\hline Utilities & $14.62 \%$ & $11.20 \%$ & $11.84 \%$ & $11.55 \%$ & $21.39 \%$ & $19.37 \%$ & $22.16 \%$ & $19.99 \%$ & $22.99 \%$ & $21.38 \%$ & $19.51 \%$ & $14.21 \%$ \\
\hline Mining, Construction and Chemicals & $9.85 \%$ & $18.34 \%$ & $13.97 \%$ & $10.05 \%$ & $17.69 \%$ & $14.92 \%$ & $13.45 \%$ & $7.82 \%$ & $14.08 \%$ & $7.13 \%$ & $8.23 \%$ & $5.87 \%$ \\
\hline Wholesale and Retail & $32.84 \%$ & $15.19 \%$ & $36.17 \%$ & $17.40 \%$ & $18.71 \%$ & $23.89 \%$ & $35.24 \%$ & $32.52 \%$ & $32.66 \%$ & $33.00 \%$ & $42.24 \%$ & $40.75 \%$ \\
\hline Services & $18.40 \%$ & $21.87 \%$ & $21.24 \%$ & $23.69 \%$ & $25.40 \%$ & $15.08 \%$ & $19.38 \%$ & $19.25 \%$ & $18.67 \%$ & $14.79 \%$ & $11.44 \%$ & $15.64 \%$ \\
\hline R2 & 0.9433 & 0.904 & 0.908 & 0.9736 & 0.9141 & 0.9032 & 0.8817 & 0.9009 & 0.9251 & 0.9019 & 0.96 & 0.9507 \\
\hline Tracking Error & $0.45 \%$ & $0.49 \%$ & $0.36 \%$ & $0.87 \%$ & $0.80 \%$ & $0.86 \%$ & $0.66 \%$ & $0.80 \%$ & $0.49 \%$ & $0.81 \%$ & $0.52 \%$ & $0.99 \%$ \\
\hline
\end{tabular}


Table 11

Style analysis - stage 5 (September 2 - December 31).

\begin{tabular}{|c|c|c|c|c|c|c|c|c|c|c|c|c|}
\hline & \multicolumn{2}{|c|}{ Malaysia } & \multicolumn{2}{|l|}{ Pakistan } & \multicolumn{2}{|c|}{ Saudi Arabia } & \multicolumn{2}{|l|}{ Qatar } & \multicolumn{2}{|l|}{ Kuwait } & \multicolumn{2}{|l|}{ UAE } \\
\hline & Islamic & Conv. & Islamic & Conv. & Islamic & Conv. & Islamic & Conv. & Islamic & Conv. & Islamic & Conv. \\
\hline \multicolumn{13}{|l|}{ Size } \\
\hline Large & $63.70 \%$ & $23.98 \%$ & $48.74 \%$ & $25.23 \%$ & $57.42 \%$ & $21.04 \%$ & $55.20 \%$ & $20.33 \%$ & $69.24 \%$ & $28.26 \%$ & $67.65 \%$ & $22.59 \%$ \\
\hline Mid & $17.32 \%$ & $29.69 \%$ & $24.38 \%$ & $41.38 \%$ & $25.24 \%$ & $33.80 \%$ & $21.22 \%$ & $29.97 \%$ & $11.74 \%$ & $33.87 \%$ & $14.56 \%$ & $39.26 \%$ \\
\hline Small & $18.98 \%$ & $46.33 \%$ & $26.88 \%$ & $33.39 \%$ & $17.34 \%$ & $45.16 \%$ & $23.57 \%$ & $49.70 \%$ & $19.01 \%$ & $37.86 \%$ & $17.79 \%$ & $38.15 \%$ \\
\hline $\mathrm{R} 2$ & 0.9211 & 0.868 & 0.8736 & 0.9441 & 0.9371 & 0.9142 & 0.9257 & 0.8895 & 0.9649 & 0.8905 & 0.9306 & 0.8823 \\
\hline Tracking Error & $0.69 \%$ & $0.45 \%$ & $0.77 \%$ & $0.97 \%$ & $0.39 \%$ & $0.83 \%$ & $0.51 \%$ & $0.66 \%$ & $0.55 \%$ & $0.48 \%$ & $0.93 \%$ & $0.64 \%$ \\
\hline \multicolumn{13}{|l|}{ Factor Strategies } \\
\hline Growth & $21.64 \%$ & $58.65 \%$ & $15.27 \%$ & $61.89 \%$ & $13.12 \%$ & $51.02 \%$ & $24.52 \%$ & $25.33 \%$ & $11.27 \%$ & $48.68 \%$ & $17.57 \%$ & $54.21 \%$ \\
\hline Value & $69.69 \%$ & $25.66 \%$ & $73.85 \%$ & $27.01 \%$ & $64.92 \%$ & $17.07 \%$ & $48.59 \%$ & $34.35 \%$ & $86.22 \%$ & $22.14 \%$ & $53.63 \%$ & $16.01 \%$ \\
\hline Momentum & $8.68 \%$ & $15.69 \%$ & $10.89 \%$ & $11.10 \%$ & $21.96 \%$ & $31.90 \%$ & $26.89 \%$ & $40.32 \%$ & $2.51 \%$ & $29.18 \%$ & $28.80 \%$ & $29.78 \%$ \\
\hline $\mathrm{R} 2$ & 0.8129 & 0.8912 & 0.7925 & 0.876 & 0.8028 & 0.8828 & 0.8018 & 0.7952 & 0.8185 & 0.8222 & 0.8418 & 0.8344 \\
\hline Tracking Error & $0.49 \%$ & $0.10 \%$ & $0.78 \%$ & $0.75 \%$ & $0.14 \%$ & $0.43 \%$ & $0.24 \%$ & $0.13 \%$ & $0.28 \%$ & $0.68 \%$ & $0.71 \%$ & $0.83 \%$ \\
\hline \multicolumn{13}{|l|}{ Sector } \\
\hline Manufacturing & $23.92 \%$ & $32.90 \%$ & $16.53 \%$ & $36.76 \%$ & $16.56 \%$ & $26.34 \%$ & $9.63 \%$ & $20.12 \%$ & $11.43 \%$ & $23.34 \%$ & $18.31 \%$ & $23.19 \%$ \\
\hline Utilities & $14.41 \%$ & $11.03 \%$ & $11.67 \%$ & $11.38 \%$ & $21.07 \%$ & $19.08 \%$ & $21.83 \%$ & $19.69 \%$ & $22.65 \%$ & $21.07 \%$ & $19.22 \%$ & $14.00 \%$ \\
\hline Mining, Construction and Chemicals & $9.70 \%$ & $18.07 \%$ & $13.77 \%$ & $9.90 \%$ & $17.43 \%$ & $14.70 \%$ & $13.25 \%$ & $7.71 \%$ & $13.87 \%$ & $7.03 \%$ & $8.11 \%$ & $5.78 \%$ \\
\hline Wholesale and Retail & $32.36 \%$ & $14.97 \%$ & $35.63 \%$ & $17.14 \%$ & $18.44 \%$ & $23.54 \%$ & $34.72 \%$ & $32.04 \%$ & $32.18 \%$ & $32.51 \%$ & $41.61 \%$ & $40.15 \%$ \\
\hline Services & $19.61 \%$ & $23.03 \%$ & $22.41 \%$ & $24.81 \%$ & $26.51 \%$ & $16.33 \%$ & $20.58 \%$ & $20.44 \%$ & $19.87 \%$ & $16.05 \%$ & $12.75 \%$ & $16.89 \%$ \\
\hline R2 & 0.906 & 0.8682 & 0.872 & 0.9351 & 0.8779 & 0.8675 & 0.8468 & 0.8652 & 0.8884 & 0.8662 & 0.922 & 0.9131 \\
\hline Tracking Error & $0.44 \%$ & $0.48 \%$ & $0.35 \%$ & $0.83 \%$ & $0.77 \%$ & $0.82 \%$ & $0.63 \%$ & $0.77 \%$ & $0.48 \%$ & $0.78 \%$ & $0.49 \%$ & $0.95 \%$ \\
\hline
\end{tabular}


Table 12

Style analysis - stage 6 (January 1 - May 31, 2021).

\begin{tabular}{|c|c|c|c|c|c|c|c|c|c|c|c|c|}
\hline & \multicolumn{2}{|c|}{ Malaysia } & \multicolumn{2}{|c|}{ Pakistan } & \multicolumn{2}{|c|}{ Saudi Arabia } & \multicolumn{2}{|l|}{ Qatar } & \multicolumn{2}{|l|}{ Kuwait } & \multicolumn{2}{|l|}{ UAE } \\
\hline & Islamic & Conv. & Islamic & Conv. & Islamic & Conv. & Islamic & Conv. & Islamic & Conv. & Islamic & Conv. \\
\hline \multicolumn{13}{|l|}{ Size } \\
\hline Large & $73.76 \%$ & $27.77 \%$ & $56.44 \%$ & $29.21 \%$ & $66.49 \%$ & $24.36 \%$ & $63.92 \%$ & $23.54 \%$ & $80.18 \%$ & $32.72 \%$ & $78.33 \%$ & $26.16 \%$ \\
\hline Mid & $20.06 \%$ & $34.38 \%$ & $28.23 \%$ & $47.92 \%$ & $29.23 \%$ & $39.14 \%$ & $24.57 \%$ & $34.70 \%$ & $13.60 \%$ & $39.22 \%$ & $16.86 \%$ & $45.46 \%$ \\
\hline Small & $6.18 \%$ & $37.86 \%$ & $15.33 \%$ & $22.87 \%$ & $4.28 \%$ & $36.50 \%$ & $11.51 \%$ & $41.76 \%$ & $6.23 \%$ & $28.05 \%$ & $4.81 \%$ & $28.39 \%$ \\
\hline $\mathrm{R} 2$ & 0.8398 & 0.7914 & 0.7965 & 0.8608 & 0.8544 & 0.8336 & 0.844 & 0.8111 & 0.8798 & 0.8119 & 0.8485 & 0.8045 \\
\hline $\begin{array}{l}\text { Tracking Error } \\
\text { Factor Strategies }\end{array}$ & $0.63 \%$ & $0.41 \%$ & $0.70 \%$ & $0.88 \%$ & $0.35 \%$ & $0.75 \%$ & $0.47 \%$ & $0.60 \%$ & $0.50 \%$ & $0.44 \%$ & $0.85 \%$ & $0.59 \%$ \\
\hline Growth & $18.69 \%$ & $50.65 \%$ & $13.19 \%$ & $53.45 \%$ & $11.33 \%$ & $44.07 \%$ & $21.17 \%$ & $21.88 \%$ & $9.73 \%$ & $42.04 \%$ & $15.17 \%$ & $46.82 \%$ \\
\hline Value & $60.18 \%$ & $22.16 \%$ & $63.78 \%$ & $23.33 \%$ & $56.07 \%$ & $14.74 \%$ & $41.96 \%$ & $29.66 \%$ & $74.46 \%$ & $19.12 \%$ & $46.31 \%$ & $13.82 \%$ \\
\hline Momentum & $21.13 \%$ & $27.19 \%$ & $23.04 \%$ & $23.22 \%$ & $32.60 \%$ & $41.19 \%$ & $36.86 \%$ & $48.46 \%$ & $15.80 \%$ & $38.84 \%$ & $38.51 \%$ & $39.36 \%$ \\
\hline $\mathrm{R} 2$ & 0.9167 & 1.0049 & 0.8936 & 0.9877 & 0.9052 & 0.9954 & 0.9041 & 0.8967 & 0.923 & 0.9272 & 0.9492 & 0.9408 \\
\hline Tracking Error & $0.56 \%$ & $0.11 \%$ & $0.88 \%$ & $0.85 \%$ & $0.16 \%$ & $0.48 \%$ & $0.27 \%$ & $0.15 \%$ & $0.32 \%$ & $0.77 \%$ & $0.80 \%$ & $0.93 \%$ \\
\hline \multicolumn{13}{|l|}{ Sector } \\
\hline Manufacturing & $27.60 \%$ & $37.96 \%$ & $19.07 \%$ & $42.41 \%$ & $19.10 \%$ & $30.40 \%$ & $11.11 \%$ & $23.22 \%$ & $13.19 \%$ & $26.93 \%$ & $21.13 \%$ & $26.75 \%$ \\
\hline Utilities & $16.62 \%$ & $12.73 \%$ & $13.46 \%$ & $13.14 \%$ & $24.31 \%$ & $22.02 \%$ & $25.19 \%$ & $22.72 \%$ & $26.13 \%$ & $24.31 \%$ & $22.18 \%$ & $16.15 \%$ \\
\hline Mining, Construction and Chemicals & $11.19 \%$ & $20.85 \%$ & $15.89 \%$ & $11.42 \%$ & $20.11 \%$ & $16.96 \%$ & $15.29 \%$ & $8.89 \%$ & $16.00 \%$ & $8.11 \%$ & $9.36 \%$ & $6.67 \%$ \\
\hline Wholesale and Retail & $37.34 \%$ & $17.27 \%$ & $41.11 \%$ & $19.78 \%$ & $21.27 \%$ & $27.16 \%$ & $40.06 \%$ & $36.97 \%$ & $37.13 \%$ & $37.52 \%$ & $46.19 \%$ & $46.33 \%$ \\
\hline Services & $7.24 \%$ & $11.19 \%$ & $10.47 \%$ & $13.25 \%$ & $15.20 \%$ & $3.46 \%$ & $8.36 \%$ & $8.20 \%$ & $7.55 \%$ & $3.14 \%$ & $1.16 \%$ & $4.10 \%$ \\
\hline R2 & 0.8976 & 0.8601 & 0.864 & 0.9264 & 0.8697 & 0.8594 & 0.839 & 0.8572 & 0.8802 & 0.8582 & 0.9134 & 0.9046 \\
\hline Tracking Error & $0.43 \%$ & $0.47 \%$ & $0.35 \%$ & $0.83 \%$ & $0.76 \%$ & $0.82 \%$ & $0.62 \%$ & $0.76 \%$ & $0.47 \%$ & $0.77 \%$ & $0.49 \%$ & $0.94 \%$ \\
\hline
\end{tabular}


Table 13

Volatility timing during the pandemic.

\begin{tabular}{|c|c|c|c|c|c|c|c|c|c|c|c|c|c|c|}
\hline \multicolumn{15}{|c|}{ Islamic Funds } \\
\hline & $\Upsilon_{\text {im }}$ Full Period & & $\Upsilon_{\text {im }}$ Stage 1 & & $\Upsilon_{\text {im }}$ Stage 2 & & $\Upsilon_{\mathrm{im}}$ Stage 3 & & $\Upsilon_{\text {im }}$ Stage 4 & & $\Upsilon_{\text {im }}$ Stage 5 & & $\Upsilon_{\text {im }}$ Stage 6 & \\
\hline Malaysia & -0.0869 & $* *$ & -0.0837 & $* * *$ & -0.0944 & *** & -0.1659 & $* * *$ & -0.0926 & $* *$ & -0.0937 & $* * *$ & -0.1023 & $* *$ \\
\hline Pakistan & -0.0366 & $* *$ & -0.0277 & $* * *$ & -0.0512 & $* * *$ & -0.0515 & $* * *$ & -0.0502 & $* *$ & -0.0508 & ** & -0.0555 & $* * *$ \\
\hline Saudi Arabia & -0.0573 & $* * *$ & -0.0512 & $* *$ & -0.0581 & $* *$ & -0.0666 & $* * *$ & -0.0569 & $* *$ & -0.0576 & $* *$ & -0.0629 & $* *$ \\
\hline Qatar & -0.0219 & $* *$ & -0.0185 & $* *$ & -0.0460 & $* *$ & -0.0177 & $* *$ & -0.0451 & $* *$ & -0.0456 & $* * * *$ & -0.0498 & $* *$ \\
\hline Kuwait & -0.0780 & $* *$ & -0.0729 & $* *$ & -0.0886 & $* *$ & -0.0946 & $* *$ & -0.0868 & $* *$ & -0.0879 & $* * * *$ & -0.0959 & $* *$ \\
\hline UAE & -0.0612 & $* *$ & -0.0468 & $* * *$ & -0.0393 & $* *$ & -0.0695 & $* * *$ & -0.0385 & $* * *$ & -0.0390 & $* * *$ & -0.0426 & $* *$ \\
\hline \multicolumn{15}{|c|}{ Conventional Funds } \\
\hline & $\Upsilon_{\mathrm{im}}$ Full Period & & $\Upsilon_{\text {im }}$ Stage 1 & & $\Upsilon_{\text {im }}$ Stage 2 & & $\Upsilon_{\text {im }}$ Stage 3 & & $\Upsilon_{\text {im }}$ Stage 4 & & $\Upsilon_{\text {im }}$ Stage 5 & & $\Upsilon_{\text {im }}$ Stage 6 & \\
\hline Malaysia & 0.0814 & $* * *$ & 0.0621 & $* *$ & 0.0638 & $* * *$ & -0.0311 & * & 0.0625 & * & 0.0633 & * & 0.0691 & * \\
\hline Pakistan & 0.0679 & * & 0.0548 & $* *$ & 0.0737 & $* *$ & 0.0206 & $* *$ & 0.0723 & $* *$ & 0.0732 & * & 0.0799 & $* *$ \\
\hline Saudi Arabia & 0.0622 & * & 0.0935 & $* *$ & 0.0925 & * & -0.0161 & $* *$ & 0.0907 & $*$ & 0.0918 & * & 0.1001 & $* *$ \\
\hline Qatar & 0.0813 & * & 0.0668 & * & 0.0884 & $* * *$ & -0.0158 & $* * *$ & 0.0867 & $*$ & 0.0877 & * & 0.0958 & $* *$ \\
\hline Kuwait & 0.0504 & $* * *$ & 0.0848 & $* * *$ & 0.0488 & $* *$ & -0.0080 & ** & 0.0479 & $* *$ & 0.0485 & $* *$ & 0.0529 & * \\
\hline UAE & 0.0936 & $* *$ & 0.0781 & $* *$ & 0.0281 & * & -0.0108 & $* *$ & 0.0276 & * & 0.0279 & $* *$ & 0.0305 & $* *$ \\
\hline
\end{tabular}

$* * *$ represent significance at $1 \%, * *$ at $5 \%$ and $*$ at $10 \%$. 
ratios compared to stage 2 . The UAE funds continue to dominate this stage with maximum adjusted Sharpe and Sortino ratios of 0.14 and 0.10 , respectively. We see a recovery in the performance of the funds from Saudi Arabia, Qatar, and Kuwait with positive riskadjusted performance among the conventional category. The Malaysian and Pakistan's funds continue to struggle in the negative zone. Jensen's alpha also supports these findings with significantly higher alphas for Islamic funds than their conventional peers. Our consolidated and stage-wise results suggest an all-around superior performance of Islamic equity funds during COVID-19.

From stages 4-6, we report the persistent performance of Islamic funds. Across all the indicators, we observe a recovery in both conventional and Islamic funds, although Islamic funds continue to dominate. The overall improvement is plausible as six months into the health crisis, the markets adapted to the new normal, and various asset classes were reverting to their fair values. The same was true for the emerging markets, and as noted by Feyen et al. (2021), most of them introduced policy responses that helped reduce the economic pressures. However, even during the later stages, the dominance of Islamic funds signifies their investment lucrativeness throughout the pandemic.

The results on stochastic dominance for SD1 to SD4 and across all countries are presented in Table 5. These are based on a 5\% statistical inference Guo et al. (2013). The findings suggest that for the entire COVID-19 period, Islamic funds dominate their conventional counterparts. This is consistent with the four orders as well as for all the countries. During stage 1 , we observe a similar pattern except for Malaysia and the UAE. In these two countries, conventional funds demonstrate superior performance utility. However, for stages 2 and 3, the observations are similar to the entire period, with Islamic funds stochastically dominating traditional equity funds. After that, for stage 5, we report a dominance of conventional funds in Qatar. While we see some anomalous observations for stages 1 and 5, it is stage 2, 3, and 4 where the impact of COVID-19 was more profound. Therefore, dominance during these specific periods reflects positively on the resilience of Islamic funds.

Table 6a presents statistics on ARCH LM that justify the use of GARCH (1,1) for estimating equations (5) and (6) (Hansen \& Lunde, 2005). The results for GARCH $(1,1)$ based CARs are also reported in Table 6a. During the pre-COVID period, we observe positive CARs for both Islamic and conventional funds. It is interesting to note that during this period, traditional funds from Malaysia, Pakistan, Saudi Arabia, and the UAE have higher CARs, while Islamic funds in Qatar and Kuwait demonstrated superior CARs compared to their counterparts. However, this completely changed during the outbreak, with conventional funds showing negative CARs across all countries for the entire period. During this time, the abnormal returns for Islamic funds remained positive. The results are similar for the six stages, with Islamic funds showing significantly positive CARs while conventional funds continue to plunge into the negative zone. Only in the UAE, we observe positive CARs for conventional funds in stage 3, but they remained lower than their Islamic peers. Finally, in stage 6, we see a reversal in conventional funds with CARs become positive despite remaining lower than Islamic funds. These results support the superior performance of Islamic funds during the turbulent times of COVID-19.

To substantiate the dependence on oil rents across countries, we report the coefficient of the oil dependence dummy in Table $6 \mathrm{~b}$. The second column shows the oil rent to GDP ratio for our sample countries, and we can see a strong dependence in Kuwait (47.3\%) and Saudi Arabia (39\%). For Qatar and the UAE, the oil rent to GDP ratio is $27.3 \%$ and 20.4\%. As noted by (M. Umar et al., 2021), the economic reliance on natural resources may influence the performance of financial institutions. However, our results demonstrate that the oil dependence dummy is not significant throughout the sample. This is consistent for the pre covid, the complete outbreak duration, and each of the six stages. Therefore, we can confirm that the economic dependence on natural resources does not influence our findings.

The stage-wise results on investment styles are presented in Tables 7-12. During stage 1, we observe a common investment trend across Islamic funds vis-à-vis the size factor. The concentration of Islamic funds across all countries is towards large firms. For example, Malaysian funds demonstrate a 45\% investment in large companies, followed by UAE and Kuwait funds with 43\% and 40\% exposure. On the contrary, the conventional funds represent higher investments in smaller companies, with Malaysian funds having a $61 \%$ concentration, followed by UAE funds that show a 55\% investment in small firms. In terms of investment strategies, most Islamic funds prefer value investments. Kuwait's funds have 55\% investment in value firms, followed by 52\% of Pakistan's funds. A notable exception among Islamic funds is in Saudi Arabia, where fund managers prefer a momentum strategy with $44 \%$ investment in winning firms.

On the contrary, the conventional funds focus on growth firms with Malaysian and Pakistan's funds, investing approximately 55\% of their portfolio in growth stocks. During stage 1, the sectoral concentration is similar for Islamic and conventional funds, with most of their investments channeled towards manufacturing, wholesale, and retail. We report minimal tracking error across size, factors, and sectoral specifications, demonstrating our findings' robustness. The results of stages 2 and 3 signify a variation in investment styles for Islamic funds, while we do not observe a significant drift for conventional funds. The Islamic funds reduced their exposure in small firms and strengthened their concentration in large firms. The Malaysian funds increased their investment in large firms to $54 \%$ in stage and $67 \%$ during stage 3 . A similar increase is witnessed in UAE funds, with considerable firm exposure of $53 \%$ during stage 2 and $71 \%$ in stage 3 . This is conceivable because as the COVID-19 deepens, the drag on small firms increases, leading to lower portfolio performance. This continues for the later stages as well.

We observe similar transitions for factors strategies where Islamic funds continue to allocate more investment towards stable value firms while reducing their growth stocks exposure. The funds based in Kuwait represent $73.5 \%$ exposure towards value stocks in stage 2 and $78 \%$ in stage 3. With the momentum strategy's dominance during stage 1, the funds from Saudi Arabia reduced their winning exposure to $36 \%$ in stage 2 and $29 \%$ in stage 3 . We also observe a gradual decline in mining, construction, and manufacturing sectors, increasing concentration towards relatively resistant wholesale and retail. For conventional funds, we note a marginal change in size, factors, and sectoral strategies only in stage 3. This shows that Islamic fund managers are more conscious of the changing environment, and in part, their superior performance is attributed to the drift in their investment styles. These observations remain consistent for stages 4,5 , and 6 , highlighting that Islamic fund managers remained proactive throughout these turbulent times by continuously 
updating their investment exposures.

We present our results on volatility timing in Table 13. Our findings suggest that Islamic funds successfully time the volatility for the entire period and during each stage. Funds demonstrate the maximum ability of volatility timing in Malaysia, followed by those in Kuwait. The varying magnitude of the coefficient suggests that as the first wave of the COVID-19 crisis progressed from stages 1-2 and 3 , Islamic funds' volatility timing increased. This continued for stages 4, 5, and 6.

On the contrary, for conventional funds, we only observe evidence of volatility timing during stage 3 . This is possibly the reason why some of the Islamic funds in our sample have positive Jensen's alpha in stage 3. However, we could not deduce evidence of volatility timing for stages 4,5 , and 6 . Nevertheless, we believe that Islamic funds' ability to adequately time the volatility and optimizing portfolio risk resulted in their superior comparative performance against conventional funds.

Our results demonstrate that Islamic funds dominate their counterparts in terms of performance, abnormal returns, and volatility timing. While the findings are mainly concentrated around the COVID-19 outbreak, the pattern is consistent even for 2019. The dominance of Islamic funds can be attributed to multiple factors. Foremost, as highlighted by (Lesser \& Walkshäusl, 2018), the superior performance stems from the expertise of fund managers about Sharia-compliant investments in countries with influential Islamic financial environments. Furthermore, findings of (Alda, 2019), (Otero-González \& Durán-Santomil, 2021) and (K. Reddy et al., 2017) associated funds performance with the screening mechanism. This is where Islamic funds stand out from their counterparts, given the intense screening process for compliance (Peillex et al., 2019). attributed the performance of Islamic funds to their asset distribution. Our findings on style analysis confirm this, and we observe a proactive transition across sectoral allocation throughout the sample period (Boo et al., 2017). and (El-Masry et al., 2016) posit that the Islamic funds are likely to do better during the financial crisis due to lower risk. Our evidence suggests that the lower risk is a function of volatility timing and it was largely absent for conventional funds. Finally, the performance of the Islamic funds can be explained by the clientle effect (Z. Umar, 2017), especially in the short term. Therefore, investment in Islamic funds is beneficial for faith-based investors and should be considered by conventional participants as shock absorbers. This is where we would like to caution our readers about a limitation of this study. As our study is in the context of the COVID-19 outbreak, our assessment is primarily limited to equity funds. This is because, as noted by (Amar et al., 2021) and (Fry-McKibbin et al., 2021), the impact of COVID-19 was much more profound for equity markets than others, for example, fixed income (Gubareva, 2020). Therefore, a possible extension of this study can be to assess the performance of faith-based and conventional funds for other categories like money market, fixed income, hybrids, etc.

\section{Conclusion}

This paper compares Islamic and conventional equity fund performance in Malaysia, Pakistan, Saudi Arabia, Qatar, Kuwait, and the UAE during the COVID-19 pandemic. Although Islamic finance is experiencing similar challenges brought by economic pressures, social distancing measures, and travel restrictions, the Sharia products' underlying principles might help resist the crisis shock. The United Nations Development Program (UNDP) has highlighted several sharia-compliant financing instruments that could be part of the integrated pandemic response plan, including zakat (charity) and Sukuk (Islamic bonds), to help countries prepare, respond, and recover from this pandemic.

In this context, we provide a timely and relevant assessment of the core investment vehicle of Islamic finance, i.e., Islamic equity funds, by reporting novel evidence of their performance during the different stages of the COVID-19 compared to the conventional counterparts. We do this by using a multifaceted methodology to evaluate the risk-adjusted performance, abnormal returns, investment styles, and volatility timing.

Our findings show that Islamic funds across six countries outperformed their conventional counterparts. These results remained consistent for the entire period and sub-periods that we introduced to mimic the outbreak's evolution. The style analysis reveals investment drift in response to the uncertainties underlying each stage. We observe significant movement for size, factors, and sectoral considerations. The drift was from riskier styles to more prudent options. This was robust for all Islamic funds in all six countries that we considered. These results are significant for investors seeking to hedge against the pandemic risks and considering the Shariahcompliant assets.

The Islamic funds dominated explicitly during the peak months of the pandemic, which confirms the safe-haven properties of the Islamic assets. The trend continues till the very recent period considered in this paper. In contrast, conventional funds only showed an ad hoc drift during the outbreak. This asset allocation (and reallocation) explains the robust performance of Islamic fund managers. There are important implications of this particular finding. The fund managers and regulators should be conscious of the importance of fund screening which is the core differential factor of the two types of funds. The investment styles help in devising a robust strategy during periods of financial turbulence. While the pandemic is still far from over, these strategies can assist in limiting investment losses. Finally, we document the evidence of volatility timing for Islamic equity funds while it was absent for conventional funds. In short, Islamic equity funds demonstrated differentials in risk-adjusted performance, investment styles, and volatility timing compared to their conventional counterparts.

Pandemics like COVID-19 are rare, and therefore our research provides some unique comparative assessment of Islamic and conventional funds during this turbulent period. However, we would like to caution the readers about these findings' preliminary nature that is based on the data available for the seventeen months of the outbreak. Also, our inferences are drawn from equity funds only. Further research can include fixed income, money market, hybrid funds, etc., to assess the resilience of Sharia-compliant funds in the medium to long term. 


\section{Author statement}

Larisa Yarovaya: conceptualization, editing, preparing for submission.

Nawazish Mirza: conceptualization, data collection, discussion of empirical results.

Syed Kumail Abbas Rizvi: conceptualization, data collection, discussion of empirical results.

Irum Saba: data handling, data analysis; writing.

Bushra Naqvi: empirical analysis; discussion of the results; writing.

\section{References}

Al-Khazali, O., Lean, H. H., \& Samet, A. (2014). Do islamic stock indexes outperform conventional stock indexes? A stochastic dominance approach. Pacific-Basin Finance Journal, 28, 29-46. https://doi.org/10.1016/j.pacfin.2013.09.003

Alam, N., Arshad, S., \& Rizvi, S. A. R. (2016). Do islamic stock indices perform better than conventional counterparts? An empirical investigation of sectoral efficiency. Review of Financial Economics, 31, 108-114. https://doi.org/10.1016/j.rfe.2016.06.003

Alda, M. (2019). ESG fund scores in UK SRI and conventional pension funds: Are the ESG concerns of the SRI niche affecting the conventional mainstream? Finance Research Letters, 101313. https://doi.org/10.1016/j.frl.2019.101313

Amar, A. B., Belaid, F., Youssef, A., Chiao, B., \& Guesmi, K. (2021). The unprecedented reaction of equity and commodity markets to COVID-19. Finance Research Letters, 38, 101853. https://doi.org/10.1016/j.frl.2020.101853

Bellalah, M., \& Chayeh, Z. (2015). Advanced risk profile analysis of Islamic equity investment: Evidence from the American, Asian and European markets. Journal of Risk, 17(6), 73-99. https://doi.org/10.21314/JOR.2015.305

Boo, Y. L., Ee, M. S., Li, B., \& Rashid, M. (2017). Islamic or conventional mutual funds: Who has the upper hand? Evidence from Malaysia. Pacific-Basin Finance Journal, 42, 183-192. https://doi.org/10.1016/j.pacfin.2016.01.004

Busse, J. A. (1999). Volatility timing in mutual funds: Evidence from daily returns. Review of financial studies. https://doi.org/10.1093/rfs/12.5.1009

Cao, C., Iliev, P., \& Velthuis, R. (2017). Style drift: Evidence from small-cap mutual funds. Journal of Banking \& Finance, 78, 42-57. https://doi.org/10.1016/j. jbankfin.2017.01.009

Cheema, M. A., \& Nartea, G. V. (2018). Cross-sectional and time-series momentum returns: Are islamic stocks different? Applied Economics, $50(54)$, $5830-5845$. https://doi.org/10.1080/00036846.2018.1488068

Damianov, D. S., \& Elsayed, A. H. (2020). Does Bitcoin add value to global industry portfolios? Economics Letters, 191, 108935. https://doi.org/10.1016/j. econlet.2019.108935

El-Masry, A. A., de Mingo-López, D. V., Matallín-Sáez, J. C., \& Tortosa-Ausina, E. (2016). Environmental conditions, fund characteristics, and Islamic orientation: An analysis of mutual fund performance for the MENA region. Journal of Economic Behavior \& Organization, 132, 174-197. https://doi.org/10.1016/j. jebo.2016.10.015

Feyen, E., Alonso Gispert, T., Kliatskova, T., \& Mare, D. S. (2021). Financial sector policy response to COVID-19 in emerging markets and developing economies. Journal of Banking \& Finance, 106184. https://doi.org/10.1016/j.jbankfin.2021.106184

Fry-McKibbin, R., Greenwood-Nimmo, M., Hsiao, C. Y.-L., \& Qi, L. (2021). Higher-order comoment contagion among G20 equity markets during the COVID-19 pandemic. Finance Research Letters, 102150. https://doi.org/10.1016/j.frl.2021.102150

Gubareva, M. (2020). The impact of Covid-19 on liquidity of emerging market bonds. Finance Research Letters, 101826. https://doi.org/10.1016/j.frl.2020.101826

Guo, X., Zhu, X., Wong, W. K., \& Zhu, L. (2013). A note on almost stochastic dominance. Economics Letters, 121(2), 252-256. https://doi.org/10.1016/j. econlet.2013.08.020

Hansen, P. R., \& Lunde, A. (2005). A forecast comparison of volatility models: Does anything beat a GARCH(1,1)? Journal of Applied Econometrics, 20(7), 873-889. https://doi.org/10.1002/jae.800

Hayat, R., \& Kraeussl, R. (2011). Risk and return characteristics of Islamic equity funds. Emerging Markets Review, 12(2), 189-203. https://doi.org/10.1016/j. ememar.2011.02.002

Hoepner, A. G. F., Rammal, H. G., \& Rezec, M. (2011). Islamic mutual funds' financial performance and international investment style: Evidence from 20 countries. The European Journal of Finance, 17(9-10), 829-850. https://doi.org/10.1080/1351847X.2010.538521

Hsu, C. C., \& Chen, M. L. (2017). The timing of low-volatility strategy. Finance Research Letters, 23, 114-120. https://doi.org/10.1016/j.frl.2017.05.014

Jensen, M. C. (1968). The performance of mutual funds in the period 1945-1964. The Journal of Finance. https://doi.org/10.2307/2325404

Kaiser, L. (2020). ESG integration: Value, growth and momentum. Journal of Asset Management, 21(1), 32-51. https://doi.org/10.1057/s41260-019-00148-y

Lesser, K., \& Walkshäusl, C. (2018). International islamic funds. Review of Financial Economics, 36(1). https://doi.org/10.1016/j.rfe.2017.09.003

Moreira, A., \& Muir, T. (2019). Should long-term investors time volatility? Journal of Financial Economics, 131(3), 507-527. https://doi.org/10.1016/j. jfineco.2018.09.011

Otero-González, L., \& Durán-Santomil, P. (2021). Is quantitative and qualitative information relevant for choosing mutual funds? Journal of Business Research, 123, 476-488. https://doi.org/10.1016/j.jbusres.2020.10.015

Parikh, H. (2019). Emerging market equity benchmarks for Japanese investors: Countries, sectors or styles? Journal of Asset Management, 20(4), 289-300. https://doi. org/10.1057/s41260-019-00123-7

Peillex, J., Erragragui, E., Bitar, M., \& Benlemlih, M. (2019). The contribution of market movements, asset allocation and active management to Islamic equity funds' performance. The Quarterly Review of Economics and Finance, 74, 32-38. https://doi.org/10.1016/j.qref.2018.03.013

Pezier, J., \& White, A. (2006). The relative merits of investable hedge fund indices and of funds of hedge funds in optimal passive portfolios. ICMA Centre Discussion Papers in Finance, 1-32.

Reddy, K., Mirza, N., Naqvi, B., \& Fu, M. (2017). Comparative risk adjusted performance of Islamic, socially responsible and conventional funds: Evidence from United Kingdom. Economic Modelling, 66. https://doi.org/10.1016/j.econmod.2017.07.007

Rizvi, S. K. A., Mirza, N., Naqvi, B., \& Rahat, B. (2020). Covid-19 and asset management in EU: A preliminary assessment of performance and investment styles. Journal of Asset Management, 1-11. https://doi.org/10.1057/s41260-020-00172-3

Sha, Y. (2020). The devil in the style: Mutual fund style drift, performance and common risk factors. Economic Modelling, 86, 264-273. https://doi.org/10.1016/j. econmod.2019.10.004

Sharpe, W. F. (1966). Mutual fund performance. Journal of Business. https://doi.org/10.1086/294846

Sharpe, W. (1992). Asset allocation: Management style and performance measurement. Journal of Portfolio Management, 18(2), 7-19. https://doi.org/10.3905/ jpm.1992.409394

Shen, X., Tsui, A. K., \& Zhang, Z. (2019). Volatility timing in CPF investment funds in Singapore: Do they outperform non-CPF funds? Risks. https://doi.org/10.3390/ risks7040106.

Sortino, F. A., \& Price, L. N. (1994). Performance measurement in a downside risk framework. Journal of Investing. https://doi.org/10.3905/joi.3.3.59

Tee, L. T., Kew, S. R., \& Low, S. W. (2019). Do momentum strategies perform better for Islamic stocks than for conventional stocks across market states? Economic Annals, 64(221), 107-129. https://doi.org/10.2298/EKA1921107T

Uddin, G. S., Arreola Hernandez, J., Labidi, C., Troster, V., \& Yoon, S. M. (2019). The impact of financial and economic factors on Islamic mutual fund performance: Evidence from multiple fund categories. Journal of Multinational Financial Management, 52(53), 100607. https://doi.org/10.1016/j.mulfin.2019.100607 
Uddin, G. S., Hernandez, J. A., Shahzad, S. J. H., \& Yoon, S. M. (2018). Time-varying evidence of efficiency, decoupling, and diversification of conventional and Islamic stocks. International Review of Financial Analysis, 56, 167-180. https://doi.org/10.1016/j.irfa.2018.01.008

Umar, Z. (2017). Islamic vs conventional equities in a strategic asset allocation framework. Pacific-Basin Finance Journal, 42, 1-10. https://doi.org/10.1016/j. pacfin.2015.10.006

Umar, M., Ji, X., Mirza, N., \& Rahat, B. (2021). The impact of resource curse on banking efficiency: Evidence from twelve oil producing countries. Resources Policy, 72 , 102080. https://doi.org/10.1016/j.resourpol.2021.102080

Vinod, H. D. (2004). Ranking mutual funds using unconventional utility theory and stochastic dominance. Journal of Empirical Finance, 11(3), 353-377. https://doi. org/10.1016/j.jempfin.2003.06.002

Wong, W. K., Phoon, K. F., \& Lean, H. H. (2008). Stochastic dominance analysis of Asian hedge funds. Pacific-Basin Finance Journal, 16(3), 204-223. https://doi.org/ 10.1016/j.pacfin.2007.07.001 